\title{
Superradiant, single-supermode and nonlinear regimes of short pulse free electron laser oscillators
}

\author{
P. Chaix, ${ }^{1}$ N. Piovella, ${ }^{2}$ and G. Grégoire ${ }^{1}$ \\ ${ }^{1}$ Commissariat à l'Energie Atomique, Département de Physique Théorique et Appliquée, \\ Service de Physique des Plasma et Electromagnetisme, Boîte Postale 12, 91680 Bruyères-le-Châtel, France \\ ${ }^{2}$ Dipartimento di Fisica, Università degli Studi, via Celoria 16, 20133 Milano, Italy
}

(Received 16 April 1998; revised manuscript received 1 September 1998)

\begin{abstract}
We study both small signal and nonlinear regimes of free electron laser oscillators driven by short electron bunches. This work extends and completes a previous work, focusing the analysis first on the spectrum of the eigenmodes of the linear problem (supermodes) and on the description of the weakly nonlinear regime in terms of these eigenmodes and second on the fully nonlinear dynamics. Using an orthogonality property of the supermodes, we derive expressions for the amplitudes of the fundamental and secondary supermodes and we discuss the single-supermode stable operation. Then we reconsider the superradiant regime in a quasiperfectly synchronized, high-quality optical cavity. We show that superradiance actually is an intrinsically multisupermode regime, which occurs when the spectrum is nearly degenerate. Going next to the nonlinear regime, we find the nonlinear modes of the system (stationary regimes), which appear through successive Hopf bifurcations when the linear eigenmodes become unstable. We analyze the stability of the fundamental nonlinear mode and show that it gets unstable through a new supercritical Hopf bifurcation when dissipation is decreased, giving rise to a limit cycle. Finally, we reconsider the routes to chaos, showing that although the dynamical behavior of the system depends in a complicated way on the control parameters, it can be described to a large extent by the iterations of one-dimensional maps. [S1063-651X(98)11012-7]
\end{abstract}

PACS number(s): 41.60.Cr, 42.65.Sf

\section{INTRODUCTION}

Free electrons laser (FEL) oscillators are a family of devices able to produce tunable coherent radiation from a relativistic electron beam [1]. They are made of an accelerator delivering the driving electron bunches and a wiggler generating a static spatially periodic magnetic field, inserted in an optical cavity storing the produced radiation. Successive electron pulses periodically enter the wiggler, where they copropagate with the stored optical pulse. The electrons oscillate transversally and bunch on the scale of the radiation wavelength, radiating under the action of the combined fields of this optical pulse and the wiggler. The generated wavelength is a continuous function of the operating parameters (electron incoming energy, wiggler field magnitude, etc.) so that it is intrinsically tunable. The amplifying medium is the electron bunches themselves, which are continuously renewed by the accelerator, so that FELs are expected to support high power. Tunability and high power are qualities that make FELs promising radiation sources. Furthermore, they have been proved to be able to generate very short superradiant optical pulses [2]. However, the price for this versatility of FELs is their tendency to develop secondary instabilities leading to unsteady radiation output: spiking in long pulse FELs [3-5] and limit cycles in short pulse FELs [6]. The gain, efficiency, and stability of FELs depend on two types of effects: dynamical effects due to the particle/ radiation interaction, responsible for bunching and trapping the electrons, and geometrical effects due to the transport of the radiation with respect to the electrons (slippage or diffraction). In particular, the longitudinal overlapping effects dominate the FEL dynamics when the electron pulses are shorter than the slippage length $\lambda N_{w}$, which is the length overtaken by radiation at a wavelength $\lambda$ over the electrons after $N_{w}$ wiggler periods. A high gain FEL amplifier driven by electron pulses shorter than the slippage operates in the superradiant regime $[7,8]$, with the emission of intense and short radiation pulses. The slippage effect is more complex in a FEL oscillator, where the radiation, reflected by mirrors back to the wiggler entrance, interacts many times with new electron pulses periodically injected in the cavity. In fact, by varying the synchronism between the periodic beam injection and the round-trip time of the radiation in the cavity, it is possible to control the overlapping between the radiation and the electron pulses during many round-trips [9]. The transport mechanism induced by cavity detuning and the losses introduced by the cavity make the FEL oscillator driven by short electron pulses an interesting example of dissipative, nonlinear system exhibiting a large variety of nonlinear behaviors such as limit cycles, chaos, and superradiance $[10,11]$. A number of currently existing FEL oscillators in the infrared range are driven by electron pulses shorter than the slippage length. In particular, the the free-electron laser for infrared experiment (FELIX) experiment already showed both limit cycles [6] and superradiance [2].

The aim of this paper is to present analytical results on the theory of short pulse FEL oscillators, within the framework already introduced in $[11,12]$. These results contribute to the analysis of the short pulse FEL instabilities and their fully nonlinear behavior to prepare ways of using and controlling the various possible dynamical behaviors of these systems [13]. We briefly recall our model in Sec. II. In Sec. III we reconsider the linear eigenvalue problem and the "orthogonality" properties of the eigenmodes with a method giving a global view of the discrete spectrum. The limit of small cav- 
ity detuning is investigated in detail, showing how all the eigenvalues with positive gain (supermodes [14]) converge toward a common value. Explicit analytical expressions for the eigenvalues and the eigenfunctions are obtained. Then, in Sec. IV, describing the nonlinear solution in terms of supermodes, we calculate up to third order the radiation intensity and the frequency shift in the weakly nonlinear regime dominated by a single supermode. The numerical determination of the exact amplitudes of the fundamental and secondary supermodes at saturation allows us to test the domain of validity of the single-supermode regime and the third-order theory. In Sec. V we demonstrate that the superradiant regime calculated previously in the frame of an inappropriately called "single-supermode approximation" [11] in the limit of small cavity detuning actually is a multisupermode regime that occurs in the limit where all the supermodes converge toward a unique degenerate supermode. This resolves the previous paradox between the observation of superradiance both in the single-supermode model of Ref. [11] and in the transient evolution of a perfectly synchronized, lossless oscillator [15]. The second part of the paper deals with the fully nonlinear regime. Section VI gives results about the nonlinear modes, which correspond to the stationary regimes. In Sec. VII we reconsider the weakly nonlinear regime and show the nature of the limit cycle instability. In Sec. VIII we reconsider the route from the stable stationary asymptotic regime to chaos, showing that the main features of the dynamics can be described by the iterations of onedimensional return maps. Finally, conclusions are drawn in Sec. IX.

\section{MODEL}

In a short pulse FEL oscillator, an optical pulse circulates in a cavity between two reflecting mirrors and interacts at each pass with a new electron pulse along the $z$ axis of a $\lambda_{w} N_{w}$ long wiggler. The electron pulses delivered by the accelerator have an effective length $L_{b}=c Q / I_{b}$, where $Q$ is the pulse charge and $I_{b}$ is the peak current, considered much smaller than the slippage length $L_{s}=\lambda N_{w}$ (the distance of which the light overtakes the electrons while traveling along the wiggler). Following Ref. [11], we describe the optical field in the cavity by its complex slowly varying envelope $A(x, \tau)$ at the wiggler entrance, where $x=(c t-z) / L_{s}$ is the position within the optical pulse in units of the slippage length, $z$ is the position along the common wiggler, beam, and cavity axis, and $\tau=g n$ is the dimensionless coarsegrained cavity time, with $n$ the cavity round-trip number, and $g=\left(L_{b} / L_{s}\right) g_{0}$, where $g_{0}$ is the usual cw small gain coefficient [1], $g_{0}=4 \pi\left(N_{w} / \gamma_{0}\right)^{3}\left(I_{b} f / I_{0}\right)\left(a_{w} \lambda_{w} F / r_{b}\right)^{2}, \gamma_{0}$ is the beam energy in rest mass units $m c^{2}, a_{w}$ is the rms wiggler parameter, $F$ is 1 for a helical wiggler and $J_{0}(\xi)-J_{1}(\xi)$, with $\xi=a_{w}^{2} / 2\left(1+a_{w}^{2}\right)$ for a planar wiggler, $r_{b}$ is the beam radius, $I_{0}=4 \pi \epsilon_{0} m c^{3} / e \sim 17000 \mathrm{~A}$ is the Alfvén limit current, and $f$ is the filling factor describing the transverse overlap between the optical and electron pulses.

In the small gain approximation $g \ll 1$ and for short electron pulses $L_{b} \ll L_{s}$, the evolution of the optical pulse is described by the following model, whose derivation is discussed in detail in Ref. [11]:

$$
\begin{gathered}
\partial_{\tau} A=\nu \partial_{x} A+\eta\langle\exp [-i \theta]\rangle-(\alpha / 2) A, \\
\partial_{x}^{2} \theta=-\{A \exp [i \theta]+\text { c.c. }\},
\end{gathered}
$$

where $\eta(x)=1$ for $0<x<1$ and $\eta(x)=0$ elsewhere, and $A$ is such that $|A|^{2}=4 \pi N_{w} g_{0} P / P_{e}$, with $P$ the intracavity optical power and $P_{e}=m c^{2} \gamma_{0}\left(I_{b} / e\right)$ the electron beam power.

Since the electron pulse is much shorter than the slippage length, the electrons sweep back over the radiation pulse from $x=0$ to $x=1$ during the slippage time $L_{s} / c$. The particle dynamics follows the usual pendulum equation (2) for the phase $\theta=\left(k+k_{w}\right) z-c k t$, where $k=2 \pi / \lambda=2 k_{w} \gamma_{0}^{2} /(1$ $\left.+a_{w}^{2}\right)$ and $k_{w}=2 \pi / \lambda_{w}$. The field amplitude, driven by the electron bunching $\langle\exp (-i \theta)\rangle$ at the resonant wavelength $\lambda$, decays in the cavity at a rate $\alpha / 2=(1-\sqrt{R}) / g$, where $R$ is the total reflection coefficient of the mirrors. It also drifts from pass to pass due to the cavity detuning $\nu$ $=2(\delta L) / L_{s} g$, where $\delta L$ is the cavity shortening relative to the vacuum synchronism between the electrons and the reflected optical pulses. A positive cavity shortening $\nu$ is necessary to compensate for the lethargy, i.e., the tendency of the optical pulse to move, in the small signal regime, slower than in the vacuum, due to the interaction with the electrons [9].

The evolution of the system can be approximately described by a reduced number of macroscopic variables, assuming an appropriate truncation in the infinite momentum hierarchy [16]. Introducing $B=\langle\exp (-i \theta)\rangle, \quad P$ $=\langle p \exp (-i \theta)\rangle, \quad Q=\langle p\rangle, \quad$ and $\quad S=\left\langle p^{2}\right\rangle$, where $p=\partial_{x} \theta=4 \pi N_{w}\left(\gamma-\gamma_{0}\right) / \gamma_{0}$, Eqs. (1) and (2) are approximated by the following reduced model for $0<x<1$ :

$$
\begin{gathered}
\partial_{\tau} A=\left(\nu \partial_{x}-\alpha / 2\right) A+B, \\
\partial_{x} B=-i P, \\
\partial_{x} P=-A-i S B-2 i Q P+2 i Q^{2} B, \\
\partial_{x} Q=-\left[A B^{*}+\text { c.c. }\right], \\
\partial_{x} S=-2\left[A P^{*}+\text { c.c. }\right] .
\end{gathered}
$$

Combining Eqs. (3) and (6), we write the equation for the energy balance

$$
\partial_{x} Q+\left(\partial_{\tau}-\nu \partial_{x}\right)|A|^{2}=-\alpha|A|^{2} .
$$

We assume an initial cold electron beam $Q(0, \tau)=S(0, \tau)$ $=0$, without prebunching $B(0, \tau)=P(0, \tau)=0$, and a small uniform seed at the first pass $A(x, 0)=A_{0}$, simulating the spontaneous emission responsible for start-up. An analysis of the prebunching effect is presented in Ref. [17].

When the cavity is longer than the perfect synchronism length, i.e., when $\nu<0$, the radiation is retarded from pass to pass and drifts in the positive $x$ direction, leaving the interaction region through the boundary $x=1$. Then the condition $A(0, \tau)=0$ is assigned at the same boundary $x=0$ for the electronic variables, so that there is no possible gain. Conversely, when the cavity is shorter than perfect synchronism, i.e., when $\nu>0$, the radiation is advanced and drifts toward the electrons, along the negative $x$ direction, leaving the interaction region through the boundary $x=0$. Then the condi- 
tion $A(1, \tau)=0$ is assigned at the boundary $x=1$, whereas the electronic variables have their boundary conditions at $x$ $=0$. In this case an absolute instability takes place, where the backward propagation of the radiation provides the necessary feedback for a stationary gain [18]. For a perfectly synchronized cavity $\nu=0$, a transient, nonexponential growth of the radiation intensity takes place [15]. Indeed, as we will see later in more detail, the limit $\nu \rightarrow 0$ is singular [the transport term proportional to $\partial_{x} A$ in Eq. (1) disappears at $\left.\nu=0\right]$. The optical field profile at small $\nu$ can be analyzed as a boundary layer [17].

For $\nu>0$, the field drifts out of the region $0<x<1$ of $\Delta x=\nu \tau$ through $x=0$, decaying at a rate given by the cavity losses $\alpha: A(x<0, \tau)=\exp (\alpha x / 2 \nu) A(0, \tau+x / \nu)$. We define the dimensionless radiation energy

$$
\begin{aligned}
\mathcal{E}(\tau)= & \int_{-\nu \tau}^{1} d x|A(x, \tau)|^{2}=\int_{0}^{1} d x|A(x, \tau)|^{2} \\
& +\nu \int_{0}^{\tau} d \tau^{\prime}\left|A\left(0, \tau^{\prime}\right)\right|^{2} e^{-\alpha\left(\tau-\tau^{\prime}\right)} .
\end{aligned}
$$

The efficiency is obtained by integrating Eq. (8) on $x$ and using Eq. (9):

$$
\eta(\tau) \equiv-\frac{Q(1, \tau)}{4 \pi N_{w}}=\frac{1}{4 \pi N_{w}}\left(\frac{d}{d \tau}+\alpha\right) \mathcal{E}(\tau) .
$$

In the form of Eqs. (3)-(7), the model has been rescaled so that it depends only on two dimensionless operating parameters $\alpha$ (dissipation) and $\nu$ (transport). Both $\alpha$ and $\nu$ are inversely proportional to the electron pulse charge and are easily controllable experimentally by varying the cavity length and the outcoupling. Note that the transport also induces dissipation since it steadily drives a part of the radiation out of the interaction region $x \in[0,1]$. We note that the use of the reduced model (3)-(7) instead of the multiparticle equations (1) and (2) is essentially motivated by computer time saving and easier analytical treatment. It has been shown [16] that the reduced model gives the necessary qualitative behavior to discuss the main physical aspects of the problem.

It is noteworthy that the system is invariant under a global phase shift: Indeed, we consider the dynamics of the optical field envelope and the incoming electrons beam is supposed to be unbunched $[B(x=0)=0]$. It is therefore natural that the dynamics does not depend on the choice of the phase origin. The situation would be different with a prebunched electron beam $[B(x=0) \neq 0]$ : Then the relative phase between the optical field and the initial bunching factor would play a role. One may go one step further: Our system is not invariant under a time-dependent phase shift. For example, the imaginary part of the eigenvalue of an eigenmode has a definite value and represents the phase drift of the optical field from pass to pass in the optical cavity in the linear regime of Sec. III. However, our system is "covariant" under the transformations

$$
\begin{aligned}
& A(x, \tau) \rightarrow e^{i \phi(\tau)} A(x, \tau), \\
& B(x, \tau) \rightarrow e^{i \phi(\tau)} B(x, \tau),
\end{aligned}
$$

$$
\begin{gathered}
P(x, \tau) \rightarrow e^{i \phi(\tau)} P(x, \tau), \\
Q(x, \tau) \rightarrow Q(x, \tau), \\
S(x, \tau) \rightarrow S(x, \tau), \\
\alpha / 2 \rightarrow \alpha / 2+i d_{\tau} \phi(\tau) .
\end{gathered}
$$

The interpretation of this symmetry is as follows. Let us introduce in the optical cavity a device with the only effect of phase shifting the optical field by $\omega_{c}(n)$ at each pass $n$. Then Eq. (3) is modified to

$$
\partial_{\tau} A=\left[\nu \partial_{x}-\alpha / 2+i \omega(\tau)\right] A+B,
$$

where $\omega(\tau)$ is proportional to $\omega_{c}(n)$. Because of the covariance under Eqs. (11)-(16), the solution of the system with the phase shifting device differs from the unperturbed system only by a time-dependent phase $e^{-i \phi}$ where $d_{\tau} \phi=\omega$ : The phase shifting device does not affect the amplitude of the optical field or its phase gradient. In other words, the dynamics of the optical field amplitude and phase gradient is not coupled to the dynamics of the global phase. Writing $A(x, \tau)=\rho(x, \tau) e^{i \phi(x, \tau)}$, the relevant degrees of freedom of our system are the amplitude $\rho$ and the phase gradient $\partial_{x} \phi$.

\section{SMALL SIGNAL REGIME}

When the optical signal is small, Eqs. (3)-(7) reduce to the linear system for $0<x<1$,

$$
\begin{gathered}
\left(\partial_{\tau}-\nu \partial_{x}+\alpha / 2\right) A=B, \\
\frac{\partial B}{\partial x}=-i P, \\
\frac{\partial P}{\partial x}=-A,
\end{gathered}
$$

with the boundary condition $A(1, \tau)=0$ for $\nu>0$ and $A(0, \tau)=0$ for $\nu<0$. In this section we will characterize the spectrum of the linear regime, study the eigenstates of the problem (supermodes [14]), and give an accurate description of their behavior as a function of cavity detuning $\nu$. In particular, we will show an othogonality property of the supermodes, which will be used in Sec. IV to expand the general solution of the nonlinear problem in terms of supermodes.

We set $A(x, \tau)=\exp [(\mu-\alpha / 2) \tau] A_{\mu}(x)$ (and similarly for $B$ and $P$ ), where $\mu$ is the complex eigenvalue and $A_{\mu}(x)$ is the associated eigenfunction. Amplification occurs only when $2 \operatorname{Re} \mu>\alpha$ and the phase of each eigenfunction drifts at a constant rate $\operatorname{Im} \mu$. Integrating Eqs. (19) and (20) with boundary conditions $B_{\mu}(0)=0$ and $P_{\mu}(0)=0$ and substituting in Eq. (18), we obtain

$$
\nu \partial_{x} A_{\mu}+i \int_{0}^{x} d x^{\prime}\left(x-x^{\prime}\right) A_{\mu}\left(x^{\prime}\right)=\mu A_{\mu} .
$$

In the case $\nu<0$, i.e., for a cavity longer than the synchronism length, the boundary condition imposes $A_{\mu}(x)=0$. Hence, as we already stated, no amplification can occur for 
$\nu<0$. We will assume $\nu>0$ in the rest of the paper. The general solution of Eq. (21) is then

$$
\begin{aligned}
A_{\mu}(x)= & A_{N}\left[k_{1}^{2}\left(k_{2}-k_{3}\right) e^{-i k_{1} x}+k_{2}^{2}\left(k_{3}-k_{1}\right) e^{-i k_{2} x}\right. \\
& \left.+k_{3}^{2}\left(k_{1}-k_{2}\right) e^{-i k_{3} x}\right],
\end{aligned}
$$

where $A_{N}$ is a constant and $k_{j}$, with $j=1,2,3$, are the complex roots of the characteristic equation

$$
\nu k^{3}-i \mu k^{2}+1=0 \text {. }
$$

The eigenvalues $\mu$ are determined by the boundary condition $A_{\mu}(1)=0$, i.e., by the equation

$$
\begin{aligned}
C(\mu)= & k_{1}^{2}\left(k_{2}-k_{3}\right) e^{-i k_{1}}+k_{2}^{2}\left(k_{3}-k_{1}\right) e^{-i k_{2}} \\
& +k_{3}^{2}\left(k_{1}-k_{2}\right) e^{-i k_{3}}=0 .
\end{aligned}
$$

The spectrum is the set of zeros of the complex function $C(\mu)$ and is therefore discrete. Since $C(\mu)$ is a complicated oscillating function, the zeros must be found using a numerical algorithm. A part of the spectrum has already been found in Ref. [11] by an iterative method. For each $\nu$, we first approached numerically the minimum of the real, positive function $|C(i \omega)|$ by scanning the real argument $\omega$; this way we determined the eigenvalues with zero real part at some $\nu$, corresponding to the gain threshold. Then, varying $\nu$ from the threshold, the eigenvalue $\mu$ was determined by continuity, investigating the vicinity in the complex plane. Although this method allowed us to identify the three most unstable eigenvalues, it has several drawbacks. First, it does not allow one to find the eigenvalues whose real part does not vanish for some $\nu$. Second, it does not give a global view of the spectrum in the complex $\mu$ plane. Third, it is difficult to follow a given eigenvalue in the small $\nu$ region, where the eigenvalues get very close to each other.

We present here a different method to search the eigenvalues, which gives a global view of the spectrum at fixed $\nu$ : We calculate the complex function $C(\mu)$ and plot the contour lines $\operatorname{Re} C(\mu)=0$ and $\operatorname{Im} C(\mu)=0$ in the complex $\mu$ plane. The intersections of these lines satisfy Eq. (24) and are the desired eigenvalues. Once the global view of the spectrum at the given $\nu$ is obtained, it is possible to determine with any desired accuracy each eigenmode by a local search of the zero of $|C(\mu)|$. The reason why it is preferable to consider first separately the real and imaginary parts of $C(\mu)$ is because they change sign around their zeros, in contradistinction to the amplitude $|C(\mu)|$, which is always positive: This makes it much easier to find the contour lines $\operatorname{Re} C(\mu)=0$ and $\operatorname{Im} C(\mu)=0$ than the vanishing minima of $|C(\mu)|$. Figure 1 shows an example of the spectrum in the complex $\mu$ plane for $\nu=0.0205$ : The solid lines represent $\operatorname{Re} C(\mu)=0$, the dash-dotted lines $\operatorname{Im} C(\mu)=0$, and the intersections between the two sets of lines are the eigenvalues. The spectrum is twofold: The first set of eigenvalues has negative real parts and negative imaginary parts [19]; we number these eigenvalues with a negative index, $n$ $=-1,-2, \ldots$. The second set of eigenvalues has positive imaginary parts and real parts positive only for $0<\nu<\nu_{n}$; we number them with a positive index $n=1,2, \ldots$. Hence there are only a finite number of unstable eigenvalues for a

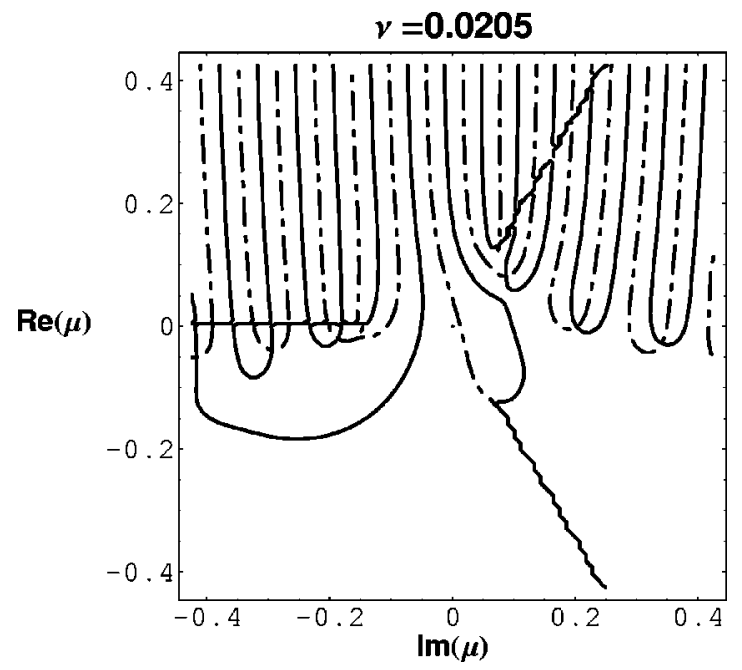

FIG. 1. Eigenvalue chart for $\nu=0.0205$. Solid line, $\operatorname{Re} C(\mu)$ $=0$; dash-dotted line, $\operatorname{Im} C(\mu)=0$. The eigenvalues are located at the intersections of the two sets of lines.

given $\nu$, but this number increases for $\nu$ decreasing toward zero (small cavity detuning or high current limit).

Figure 2 shows the behavior, as a function of $\nu$, of the real and imaginary parts of the eigenvalues $\mu_{n}$ for $n$ $=1, \ldots, 5$ (solid lines) and $n=-1,-2,-3$ (dashed lines),
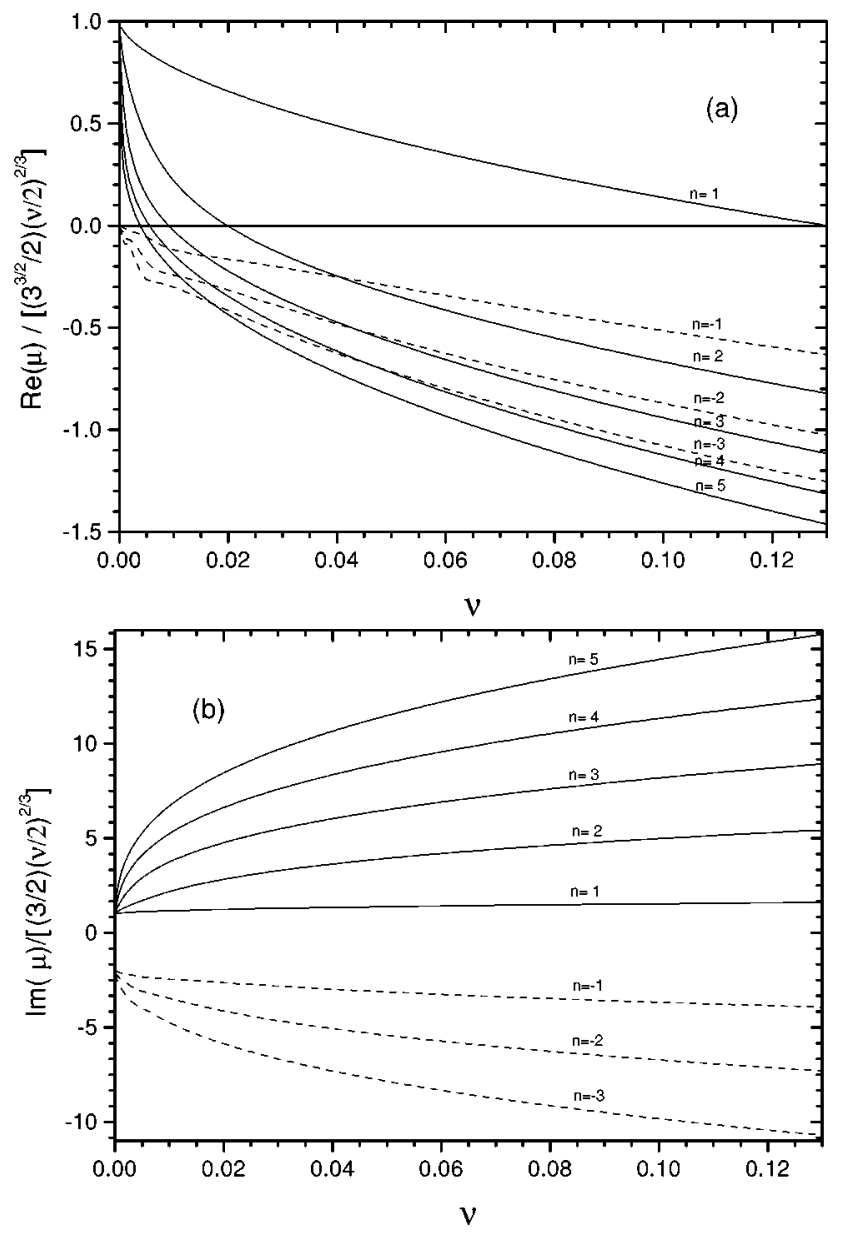

FIG. 2. (a) Real part and (b) imaginary part of the eigenvalues with a positive index (solid lines) and a negative index (dashed lines) versus the cavity detuning $\nu$. 


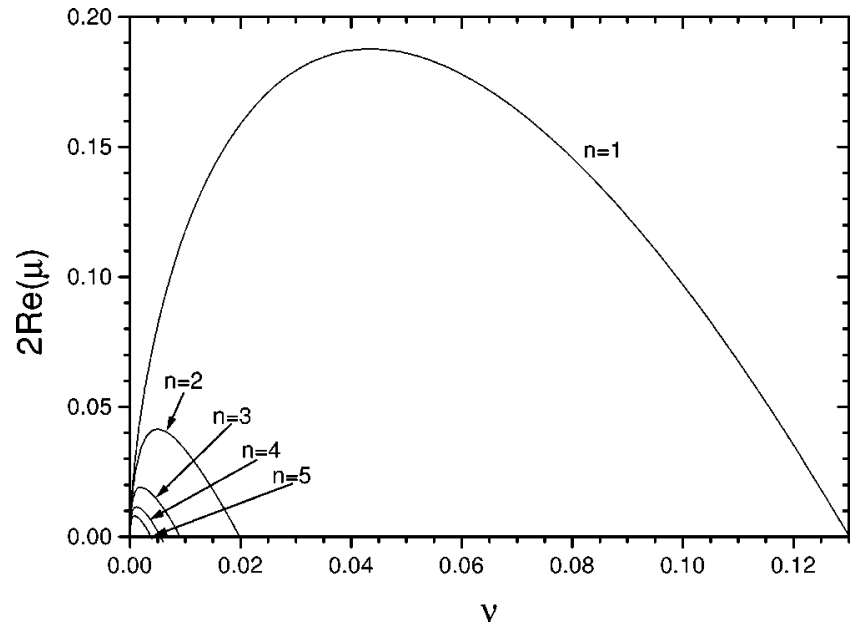

FIG. 3. Positive reduced gain $G / g=2 \operatorname{Re}(\mu)$ for the first five supermodes versus $\nu$.

scaled with (a) $\operatorname{Re}(\mu) /\left[(3 \sqrt{3} / 2)(\nu / 2)^{2 / 3}\right]$ and (b) $\operatorname{Im}(\mu) /\left[(3 / 2)(\nu / 2)^{2 / 3}\right]$. The eigenmodes with $\operatorname{Re} \mu>\alpha / 2$ will be amplified in the small signal regime. The linear dynamics is dominated by the fundamental supermode $n=1$, with a net gain of $G=g(2 \operatorname{Re} \mu-\alpha)$. Figure 3 shows the positive values of $2 \operatorname{Re} \mu$ vs $\nu$ for $n=1, \ldots, 5$ : No gain occurs for $\nu$ $>\nu_{1}=0.13$, whereas the $n=2, \ldots, 5$ eigenvalues have a positive real part for $\nu<\nu_{n}$, where $\nu_{2}=0.02, \nu_{3}=0.01, \nu_{4}$ $=0.005$, and $\nu_{5}=0.003$. We observe in Fig. 2 at small $\nu$ that the eigenvalues with $n>0$ behave like $\mu$ $=3(\nu / 2)^{2 / 3} \exp (i \pi / 6)$, whereas the eigenvalues with $n<0$ behave like $\mu=-3 i(\nu / 2)^{2 / 3}$.

The integro-differential operator defined by Eq. (21) is neither self-adjoint nor normal (it does not commute with its adjoint) and as a consequence the eigenmodes belonging to different eigenvalues are not orthogonal in the usual "energy" scalar product $(f \mid g)_{1} \equiv \int_{0}^{1} d x f(x)^{*} g(x)$. It is possible, however, to show that the supermodes $A_{n}$ satisfy the orthogonality relation [20]

$$
\left(\mu_{n}-\mu_{m}\right)\left(A_{n} \mid A_{m}\right)_{2}=0
$$

for the pseudoscalar product

$$
(f \mid g)_{2}=\int_{0}^{1} d x f(x) g(1-x),
$$

where $f$ and $g$ are two complex functions defined on the interval $[0,1]$. We note that the product $(26)$ is not a scalar product because $(f \mid g)_{2}=(g \mid f)_{2}$ and $(f \mid f)_{2}$ is not in general real and positive. Nevertheless, using the property (25), one can extract the components $a_{n}$ of any combination of supermodes: If

$$
A(x, \tau)=\sum_{n} a_{n}(\tau) A_{n}(x)
$$

then the components $a_{n}$ are

$$
a_{n}=\frac{\left(A_{n} \mid A\right)_{2}}{\left(A_{n} \mid A_{n}\right)_{2}} .
$$

We will make the assumption that the set of the supermodes $A_{n}$ is complete and write the time-dependent solutions of the nonlinear problem as a combination (27). Then the relation (28) will be used to study the time-dependent amplitudes of the solution.

In order to find an approximate solution for the eigenvalues in the case of small, positive $\nu$, we observe that for $\nu$ $=0$ the solution is identically zero. Furthermore, Eq. (24) is verified when two roots of the cubic equation coincide, e.g., $k_{2}=k_{3}$, with the solution (22) identically zero. Then we expect that, in the case of small $\nu$, all the eigenvalues will be close to the degenerate value $\mu_{0}$ for which two of the three roots of the cubic equation are equal $k_{2}=k_{3}$. This occurs for $\mu_{0}^{3}=i(27 / 4) \nu^{2}$, with $k_{2}=k_{3}=(2 i / 3 \nu) \mu_{0}$ and $k_{1}=-(i /$ $3 \nu) \mu_{0}$. Two of the three possible complex values of $\mu_{0}$, $\mu_{0}=3(\nu / 2)^{2 / 3} e^{i \pi / 6}$ and $\mu_{0}=-i 3(\nu / 2)^{2 / 3}$, respectively, are observed to be the limit for small $\nu$ of the eigenvalues with positive and negative indices, as can be observed in Fig. 2. Expanding $k$ and $\mu$ around their degenerate values $k_{0}$ $=(2 i / 3 \nu) \mu_{0}$ and $\mu_{0}$ in Eq. (23), we obtain $k_{2,3} \simeq k_{0}$ $\pm(2 i / 3 \nu) \sqrt{\mu_{0}\left(\mu-\mu_{0}\right)}+O\left(\mu-\mu_{0}\right)$. Inserting these values into the expression of the eigenfunction (22) and neglecting the nondegenerate root $k_{1}$, we obtain

$$
\begin{aligned}
A_{\mu}(x) \simeq & \left(3 A_{N} / \nu\right) e^{-i k_{0} x}\left\{e^{(2 / 3 \nu) \sqrt{\mu_{0}\left(\mu-\mu_{0}\right)}}-e^{-(2 / 3 \nu) \sqrt{\mu_{0}\left(\mu-\mu_{0}\right)}}\right. \\
& \left.+O\left(\sqrt{\left(\mu-\mu_{0}\right) / \mu_{0}}\right)\right\} .
\end{aligned}
$$

Then the condition $A_{\mu}(1)=0$ is fulfilled when $(2 / 3 \nu) \sqrt{\mu_{0}\left(\mu-\mu_{0}\right)}=i \pi n$, where $n$ is an integer, i.e., when $\mu=\mu_{0}-9 \pi^{2} n^{2} \nu^{2} / 4 \mu_{0}$, and the eigenfunctions are

$$
A_{n}(x) \simeq A_{0 n}\left[e^{-i k_{0} x} \sin n \pi x+O\left(\nu^{1 / 3}\right)\right] .
$$

Hence the eigenvalues with positive index are

$$
\mu_{n}=3(\nu / 2)^{2 / 3}\left[e^{i \pi / 6}-\pi^{2} n^{2}(\nu / 2)^{2 / 3} e^{-i \pi / 6}\right],
$$

with eigenfunctions

$$
A_{n}(x) \simeq\left(3 \sqrt{3} / n^{2} \pi^{2} \nu\right)^{1 / 2} e^{-(2 / \nu)^{1 / 3}(\sqrt{3}+i)(1-x) / 2} \sin n \pi x .
$$

The eigenvalues with a negative index are

$$
\mu_{n}=-3 i(\nu / 2)^{2 / 3}\left[1+\pi^{2} n^{2}(\nu / 2)^{2 / 3}\right],
$$

with eigenfunctions

$$
A_{n}(x)=\sqrt{2} e^{i(2 / \nu)^{1 / 3}(1-x)} \sin n \pi x .
$$

The normalization constants have been chosen in order to have

$$
\int_{0}^{1} d x\left|A_{n}(x)\right|^{2}=1 .
$$

We observe that the approximated expression (29) satisfies the orthogonality relation 


$$
\begin{aligned}
\left(A_{n} \mid A_{m}\right)_{2} & =\int_{0}^{1} d x A_{n}(1-x) A_{m}(x) \\
& =\delta_{n, m}(-1)^{n}\left(A_{0 n}^{2} / 2\right) e^{-i k_{0}} .
\end{aligned}
$$

For $\nu$ sufficiently small, the exponential factor in the expression of the supermodes (31) is large and the function is appreciably different from zero only in the trailing edge region close to $x=1$, with a width $\sigma_{x} \sim(\nu / 2)^{1 / 3}$ (boundary layer). Hence we may assume $\sin n \pi x \simeq n \pi(1-x)$ and

$$
A_{n}(x) \simeq(3 \sqrt{3} / \nu)^{1 / 2}(1-x) e^{-k(1-x)},
$$

where $k=(2 / \nu)^{1 / 3}(\sqrt{3}+i) / 2$. The associated expressions for the bunching and the momentum bunching are, respectively,

$$
\begin{gathered}
B_{n}(x) \simeq(3 \sqrt{3} / \nu)^{1 / 2}(\nu / 2)^{2 / 3} e^{i \pi / 6}(1-x+2 / k) e^{-k(1-x)} \\
P_{n}(x) \simeq(3 \sqrt{3} / \nu)^{1 / 2}(\nu / 2)^{1 / 3} e^{5 i \pi / 6}(1-x+1 / k) e^{-k(1-x)}
\end{gathered}
$$

We observe that in the limit of very small $\nu$ and using the normalization (34), the supermodes are independent of $n$. The maximum of the intensity is located at $x=1-(2 /$ $\sqrt{3})(\nu / 2)^{1 / 3}$, with $|A|_{\max }^{2}=\left(2 \sqrt{3} / e^{2}\right)(2 / \nu)^{1 / 3}$.

Figures 4 and 5 show the profiles of the supermodes $\left|A_{n}\right|$ for $n=1,3,5$ (solid line), compared with the approximated expressions (31) (dashed line) and (36) (dotted line) for $\nu$ $=2.5 \times 10^{-3}$ (Fig. 4) and $\nu=2.5 \times 10^{-4}$ (Fig. 5), together with their phase derivative $\phi^{\prime}$. The dashed line represents the approximated value $\phi^{\prime}=(1 / 2)(2 / \nu)^{1 / 3}$, derived from Eq. (31). Naturally, we observe that the expression (36), which is independent of $n$, approximates better the exact expression for smaller $\nu$ and $n$.

\section{THIRD-ORDER NONLINEAR THEORY AND SINGLE-SUPERMODE OPERATION}

We have determined the eigenmodes of the linear system (supermodes) and the complex internal product (25) for which the eigenmodes are orthogonal. In this section we use the supermodes to describe saturation by expanding in terms of supermodes the solution of an approximated model at the third order in the field amplitude. The determination of the evolution equations for the amplitudes $a_{n}(\tau)$ of the expansion (27) up to third order will allow us to characterize the weakly nonlinear regime, testing the domain of validity of the model. After deriving the exact equations for the coupled supermode amplitudes, we will focus our analysis on the single-supermode approximation, comparing the analytical result for $a_{1}$ with the value calculated from the numerical solution of Eqs. (3)-(7).

The reduced model of Eqs. (3)-(7) can be approximated in the weakly nonlinear regime retaining the nonlinear terms up to the third order in the field amplitude $A$ :

$$
\begin{aligned}
\left(\partial_{\tau}-\nu \partial_{x}+\alpha / 2\right) A= & B^{(1)}-\int_{0}^{x} d x^{\prime}\left(x-x^{\prime}\right)\left[S^{(2)} B^{(1)}\right. \\
& \left.+2 Q^{(2)} P^{(1)}\right]
\end{aligned}
$$

$$
\begin{gathered}
Q^{(2)}=-\int_{0}^{x} d x^{\prime}\left[A^{*} B^{(1)}+\text { c.c. }\right]=B^{(1)} P^{(1) *}+\text { c.c. }, \\
S^{(2)}=-2 \int_{0}^{x} d x^{\prime}\left[A^{*} P^{(1)}+\text { c.c. }\right]=2\left|P^{(1)}\right|^{2},
\end{gathered}
$$

where $B^{(1)}$ and $P^{(1)}$ are the solutions of the linear equations (19) and (20). Using Eqs. (27) and (21) in Eq. (39), we obtain

$$
\sum_{n} \dot{a}_{n} A_{n}=\sum_{n}\left(\mu_{n}-\alpha / 2\right) a_{n} A_{n}-\sum_{m, k, l} \Lambda_{m k l} a_{m} a_{k}^{*} a_{l},
$$

where the overdot stands for a derivative with respect to $\tau$,

$$
\Lambda_{m k l}=2 \int_{0}^{x} d x^{\prime}\left(x-x^{\prime}\right)\left\{P_{m} P_{k}^{*} B_{l}+P_{m} B_{k}^{*} P_{l}+B_{m} P_{k}^{*} P_{l}\right\}
$$

and we have set $B^{(1)}=\Sigma_{n} a_{n} B_{n}$ and $P^{(1)}=\Sigma_{n} a_{n} P_{n}$. We observe that $\Lambda_{m k l}=\Lambda_{l k m}$. Using the orthogonality relation (25) in Eq. (42), we obtain

$$
\dot{a}_{n}=\left(\mu_{n}-\alpha / 2\right) a_{n}-\sum_{m, k, l} \beta_{m k l}^{n} a_{m} a_{k}^{*} a_{l}
$$

where

$$
\beta_{m k l}^{n}=\frac{\left(A_{n} \mid \Lambda_{m k l}\right)_{2}}{\left(A_{n} \mid A_{n}\right)_{2}}=-i \frac{\left(B_{n} \mid \Lambda_{m k l}^{\prime \prime}\right)_{2}}{\left(A_{n} \mid A_{n}\right)_{2}}
$$

In the expression (45) we have integrated twice per part us$\operatorname{ing} A_{n}=-i B_{n}^{\prime \prime}$. Equation (44) is an exact result of the thirdorder theory and rules the temporal evolution of all the supermode amplitudes $a_{n}(\tau)$.

When the real part of the eigenvalue $n=1$ is close to the loss $\alpha / 2$, the first supermode is close to the gain threshold and the other supermodes are strongly damped. If we suppose that the component of the fundamental supermode remains always much larger than the components of the others supermodes, $\left|a_{n}\right| \ll\left|a_{1}\right| \ll 1$ for $n \geqslant 2$, then

$$
\dot{a}_{n}=\left(\mu_{n}-\alpha / 2\right) a_{n}-\beta_{n}\left|a_{1}\right|^{2} a_{1},
$$

where $\beta_{n}=\beta_{111}^{n}$. Equation (46) for $n=1$ is the usual Landau-Ginzburg equation [Eq. (42) of Ref. [11]] for the single-supermode regime, generalized for all the secondary supermodes $(n \geqslant 2)$ driven by the fundamental supermode $n=1$. Equation (46) has been obtained from the exact solution using the orthogonality relation (25). Conversely, Eq. (42) of Ref. [11] was derived assuming the single-supermode approximation before extracting the fundamental component and the saturation coefficient was calculated using the energy scalar product for which the supermodes are not orthogonal, as $\beta_{1}=\left(A_{1} \mid \Lambda_{111}\right)_{1}=\int_{0}^{1} d x A_{1}^{*}(x) \Lambda_{111}(x)$. Hence it was actually not a true single-supermode regime, but, as we will see below, a limit regime for cavity detuning close to zero, where all the supermodes tend to the same expression (36) independent of $n$. This is referred to as the superradiant re- 

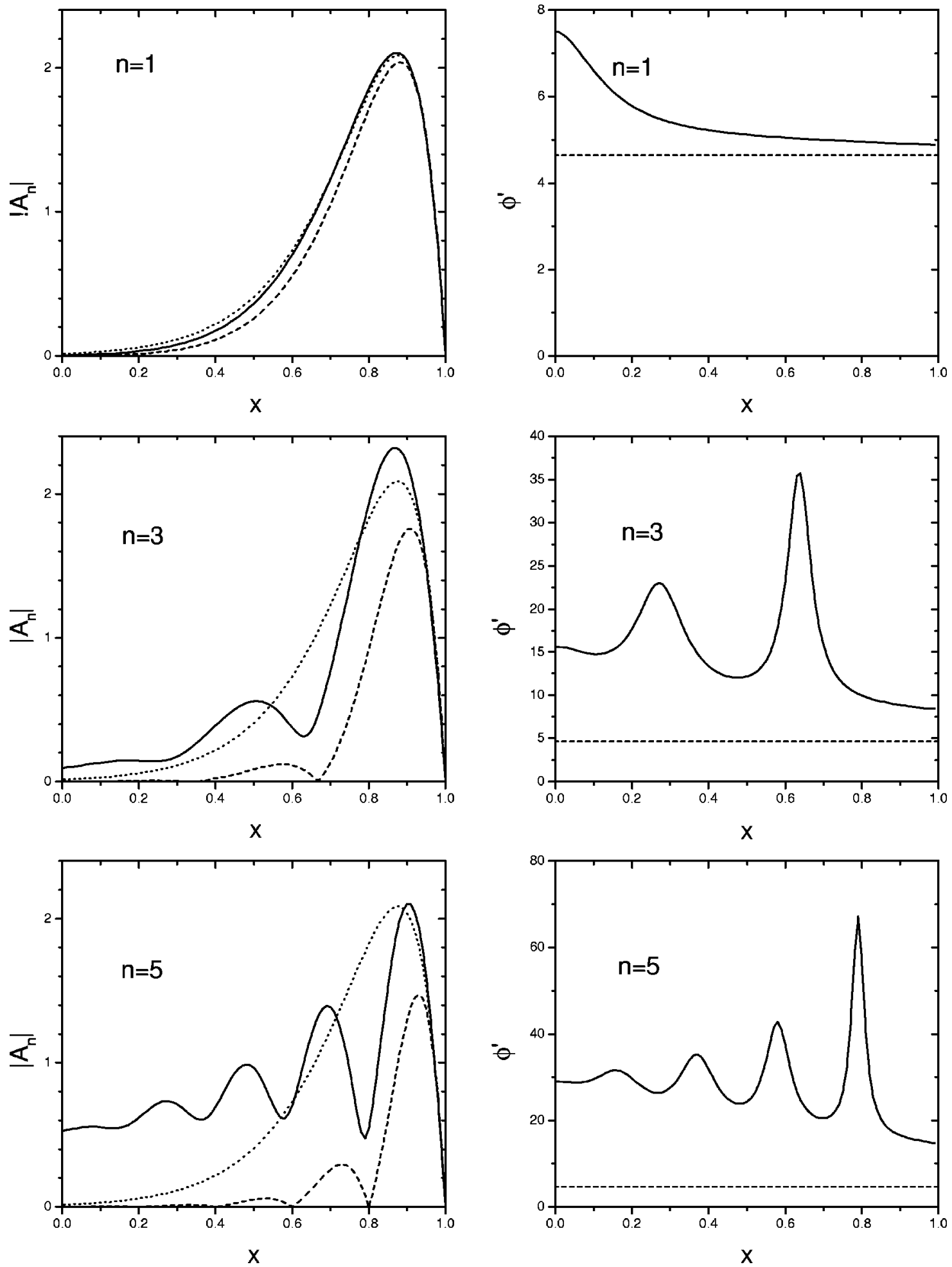

FIG. 4. Left column: supermodes profiles $\left|A_{n}(x)\right|$ (solid line) for $n=1,3,5$ and $\nu=2.5 \times 10^{-3}$; dashed lines, Eq. (31); dotted lines, Eq. (36). Right column: phase derivative $\phi^{\prime}(x)$; dashed lines, $\phi^{\prime}=(1 / 2)(2 / \nu)^{1 / 3}$.

gime, already demonstrated analytically in a perfectly synchronized, lossless FEL oscillator [15].

In the steady state, the amplitude of the fundamental supermode can be written $a_{1}=\rho_{1 s} \exp \left(i \omega_{1 s} \tau\right)$. Then Eq. (46) gives

$$
\rho_{1 s}=\sqrt{g_{1} / 2 \operatorname{Re} \beta_{1}},
$$

$$
\omega_{1 s}=\operatorname{Im}\left(\mu_{1}-\beta_{1} \rho_{1 s}^{2}\right)
$$

where

$$
g_{n}=2 \operatorname{Re} \mu_{n}-\alpha
$$

is the net gain of the $n$th supermode. In the single-supermode regime, the steady-state efficiency is therefore, from Eqs. (9) and (10), 

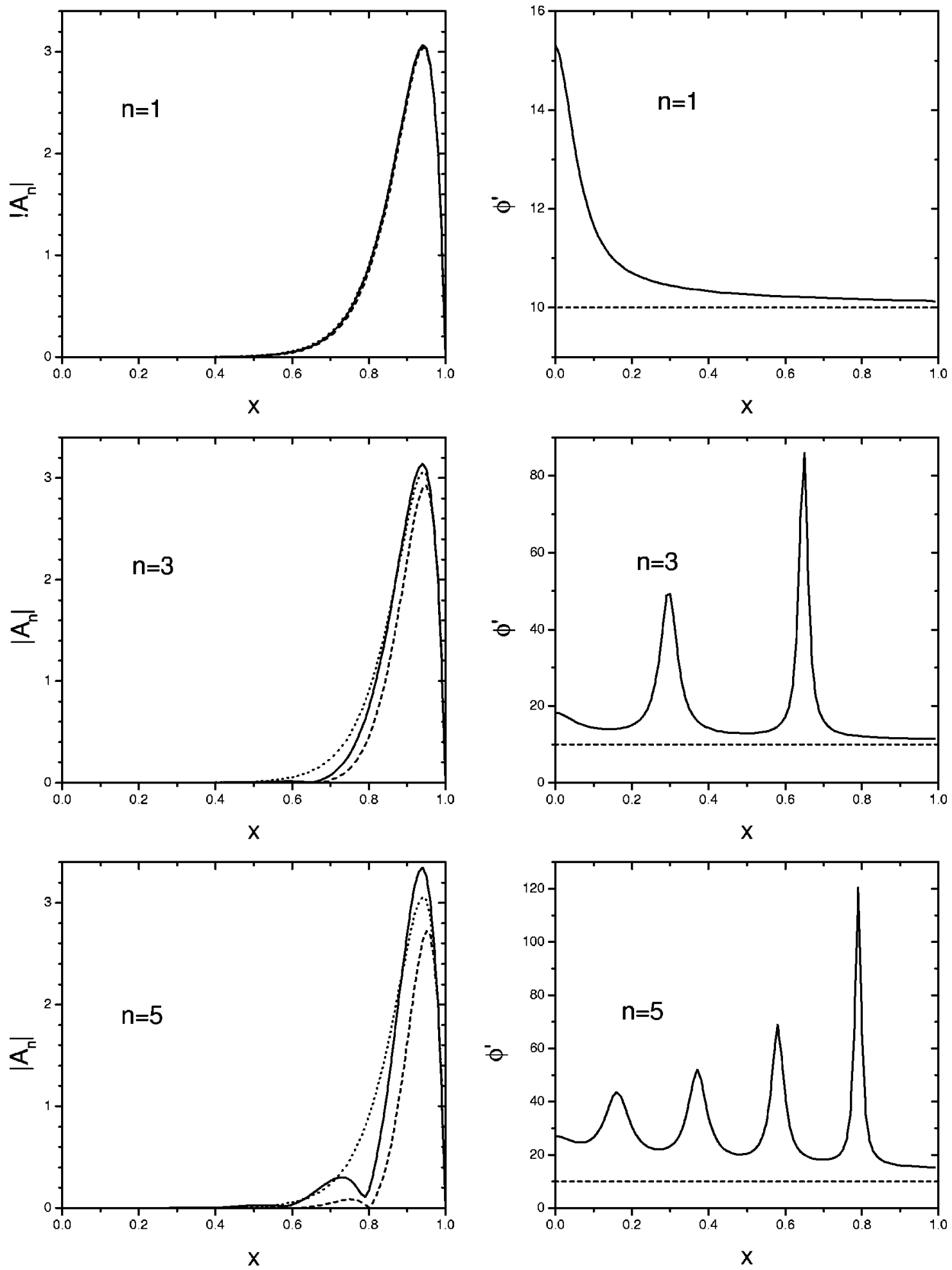

FIG. 5. Same as Fig. 4, but for $\nu=2.5 \times 10^{-4}$.

$$
\eta=\frac{\rho_{1 s}^{2}}{4 \pi N_{w}}\left[\alpha+\nu\left|A_{1}(0)\right|^{2}\right],
$$

where the second term in the square brackets is due to the radiation freely propagating in the region $-\nu \tau<x<0$. Equation (46) for $n \geqslant 2$ determines the amplitudes of the secondary supermodes driven by the fundamental in the stationary regime. Defining $a_{n}=\bar{a}_{n s} \exp \left(i \omega_{1 s} \tau\right)$ for $n \geqslant 2$, we obtain from Eq. (46)

$$
\bar{a}_{n s}=\frac{\rho_{1 s}^{3} \beta_{n}}{\mu_{n}-i \omega_{1 s}-\alpha / 2} .
$$

Figures 6-8 show $\left|a_{n}\right|$ vs $\nu$ for the first supermodes for $\alpha$ $=0.18, \alpha=0.16$, and $\alpha=0.05$, respectively, calculated using Eq. (28), i.e., by projecting the numerical solution obtained by integration of Eqs. (3)-(7) on the supermode $A_{n}$. In Figs. 6 and 7 the solid lines represent the analytical values given by Eqs. (47) and (51). We first note the agreement (and 


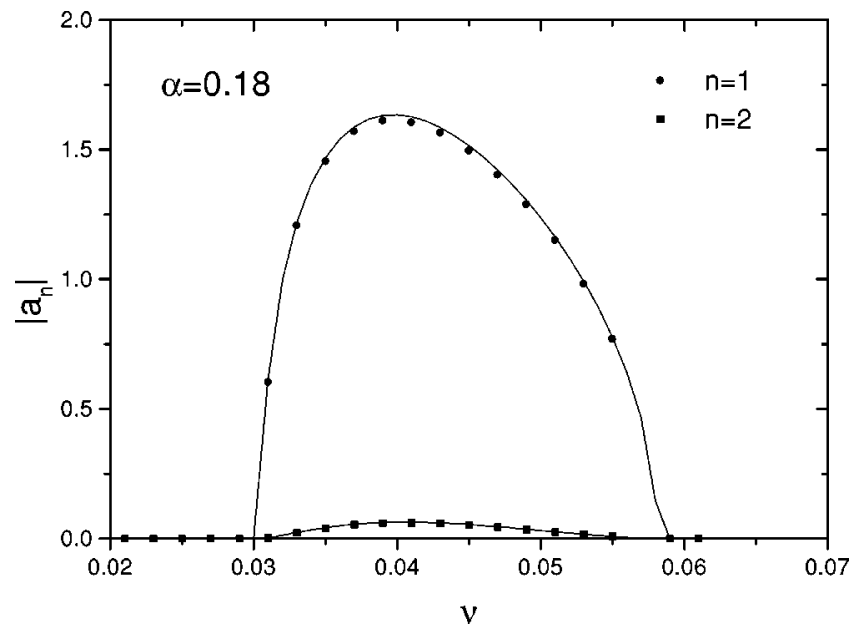

FIG. 6. $\left|a_{n}\right|$ vs $\nu$ for the supermodes $n=1$ and $n=2$ for $\alpha$ $=0.18$; solid lines, analytical values; circles and squares, Eq. (28), where $A(x, \tau)$ is the numerical solution.

therefore cross validation) between numerical simulation and third-order theory, where the condition $\left|a_{n}\right| \ll\left|a_{1}\right| \ll 1$ for $n$ $\geqslant 2$ is satisfied. We furthermore observe that the amplitude of the fundamental is much larger than the amplitudes of the other modes, so the single-supermode approximation is good. On the contrary, in Figure 8 at small $\nu$, the first two supermodes have comparable amplitudes and the singlesupermode approximation is irrelevant. Figure 9 shows the scaled efficiency $4 \pi N_{w} \eta$ vs $\nu$ for $\alpha=0.18$ and $\alpha=0.16$ as obtained from the numerical solution of Eqs. (3)-(7) (circles and squares), compared with the analytical result of Eq. (50) (solid lines).

\section{SUPERRADIANT REGIME}

We have seen that the single-supermode approximation fails near $\nu=0$ and $\alpha=0$, where the amplitudes $a_{n}$ are of the same order of magnitude and tend toward a common value. This is consistent with our results of Sec. III, where we have shown that the eigenmodes for small $\nu$ become degenerate and tend to a common expression given by Eq. (36), independent of $n$. In this limit case, we can use the explicit expressions of the eigenmodes to obtain again an analytic

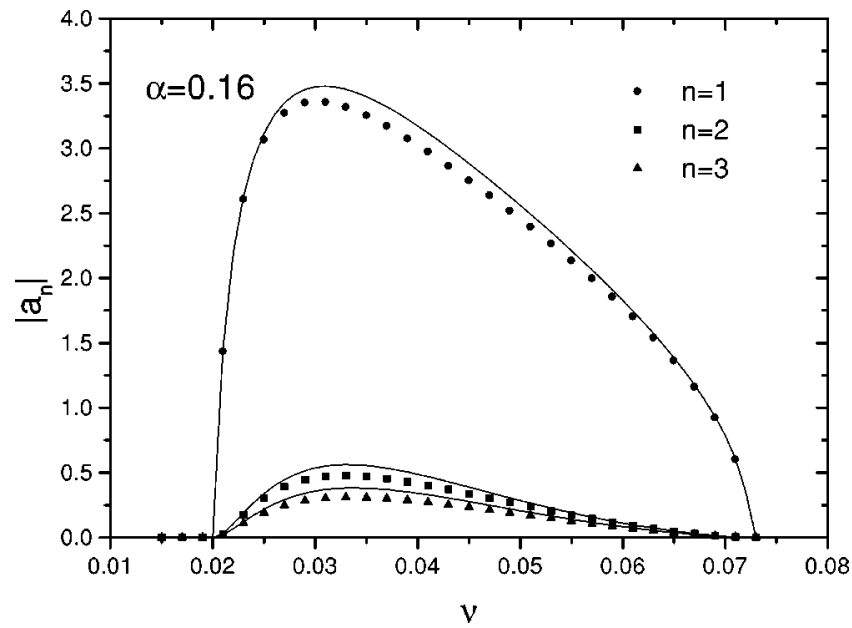

FIG. 7. Same as Fig. 6, but for $\alpha=0.16$.

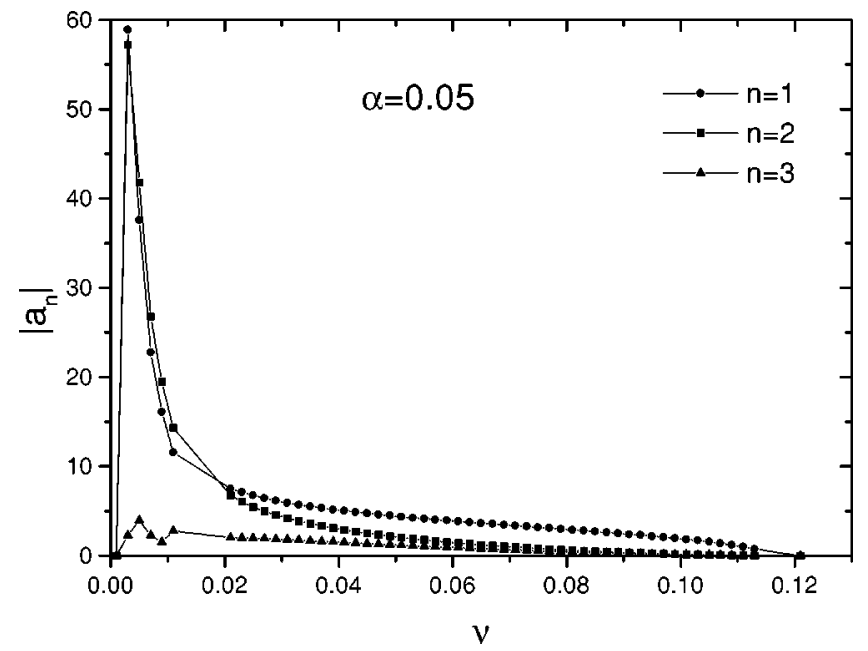

FIG. 8. $\left|a_{n}\right|$ vs $\nu$ for the supermodes $n=1, n=2$, and $n=3$ for $\alpha=0.05$, calculated using Eq. (28), where $A(x, \tau)$ is the numerical solution.

evaluation of the saturated intensity. Instead of the expansion Eq. (27), which is now useless because it would require a large number of components, we assume $A(x, \tau)$ $\simeq a_{S}(\tau) A_{S}(x)$, where $A_{S}(x)$ is the approximate solution (36), which is the common approximate shape of all the nearly degenerate supermodes. Then Eq. (44) reduces to the simpler equation

$$
\dot{a}_{S}=\left(\mu_{S}-\alpha / 2\right) a_{S}-\beta_{S}\left|a_{S}\right|^{2} a_{S},
$$

where $\mu_{S}=3(\nu / 2)^{2 / 3} e^{i \pi / 6}$,

$$
\begin{aligned}
\beta_{S}= & \int_{0}^{1} d x A_{S}(x)^{*} \Lambda_{S}(x)=i \int_{0}^{1} d x B_{S}(x) \Lambda_{S}^{\prime \prime}(x) \\
& -\left[P_{S}^{*}(1) \Lambda_{S}(1)+i B_{S}^{*}(1) \Lambda_{S}^{\prime}(1)\right],
\end{aligned}
$$

and $\Lambda_{S}$ has the same form as Eq. (43), with $B_{S}$ and $P_{S}$ given by Eqs. (37) and (38). In order to evaluate the limit of the saturation coefficient $\beta_{S}$ for small $\nu$, it is convenient to in-

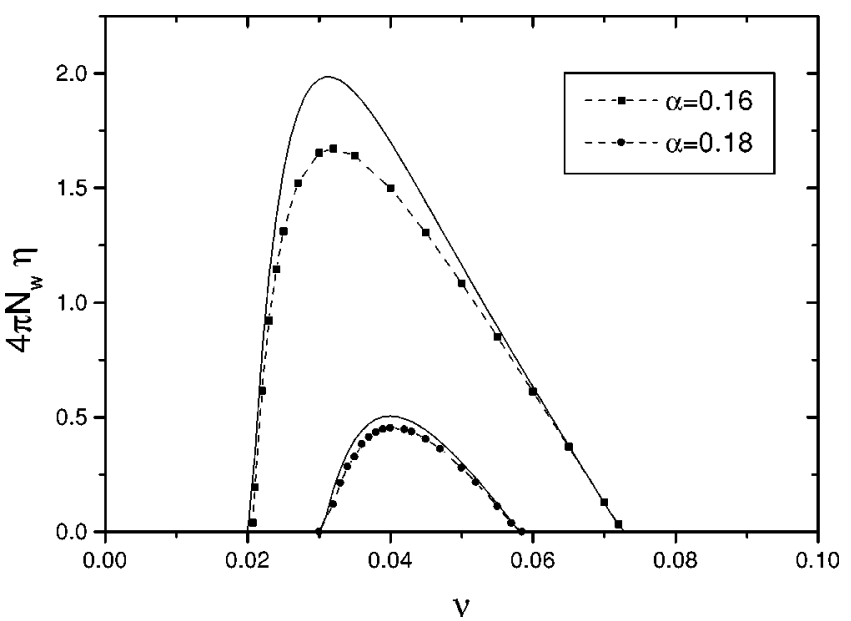

FIG. 9. Scaled efficiency $4 \pi N_{w} \eta$ vs $\nu$ for $\alpha=0.18$ and $\alpha$ $=0.16$. Solid lines, analytical solution, Eq. (50). 
troduce the new integration variable $y=(2 / \nu)^{1 / 3}(1-x)$ and extend the upper integration limit to $y=\infty$. Then Eqs. (36)(38) can be written as

$$
\begin{gathered}
A_{S}=(\nu / 2)^{-1 / 6} \widetilde{A}(y), \\
B_{S}=(\nu / 2)^{1 / 2} \widetilde{B}(y), \\
P_{S}=(\nu / 2)^{1 / 6} \widetilde{P}(y),
\end{gathered}
$$

where

$$
\begin{gathered}
\widetilde{A}(y)=(3 \sqrt{3} / 2)^{1 / 2} y e^{-c y}, \\
\widetilde{B}(y)=(3 \sqrt{3} / 2)^{1 / 2}(2+c y) e^{-c y}, \\
\widetilde{P}(y)=(3 \sqrt{3} / 2)^{1 / 2}(1+c y) e^{2 i \pi / 3} e^{-c y},
\end{gathered}
$$

and $c=e^{i \pi / 6}$. We observe that $\widetilde{A}, \widetilde{B}$, and $\widetilde{P}$ are independent of $\nu$. Using Eqs. (55)-(59) in Eq. (53), we obtain

$$
\begin{aligned}
\beta_{S} & =(\nu / 2)^{5 / 3}\left\{\int_{0}^{\infty} d y K(y)\left[i \widetilde{B}^{*}(y)-P^{*}(0) y-i B^{*}(0)\right]\right\} \\
& =(\nu / 2)^{5 / 3}[4.09-i 4.59],
\end{aligned}
$$

where

$$
K(y)=2\left[2|\widetilde{P}(y)|^{2} \widetilde{B}(y)+\widetilde{P}^{2}(y) \widetilde{B}^{*}(y)\right]
$$

and the integral in Eq. (60) has been evaluated numerically. Then the steady-state efficiency for small cavity detuning is

$$
\eta=\frac{\alpha}{4 \pi N_{w}} \frac{2 \operatorname{Re} \mu_{S}-\alpha}{2 \operatorname{Re} \beta_{S}} \simeq \frac{\alpha}{4 \pi N_{w}} \frac{3 \sqrt{3}(\nu / 2)^{2 / 3}-\alpha}{8(\nu / 2)^{5 / 3}} .
$$

The maximum efficiency $\eta_{\max } \simeq 1.43 / 4 \pi N_{w} \sqrt{\alpha}$ occurs at $\nu_{\text {opt }}=2(5 \alpha / 9 \sqrt{3})^{3 / 2} \sim 0.363 \alpha^{3 / 2}$, with a peak power $|A|_{\text {max }}^{2}$ $=1.2 / \alpha^{2}$ and a width $\sigma_{x} \sim 0.56 \sqrt{\alpha}$. Hence the optical pulse is $N_{s}=N_{w} \sigma_{x}=0.56 N_{w} \sqrt{\alpha}$ optical wavelengths long, the efficiency is $\eta=1 / 5 \pi N_{s}$, and the peak power is $P_{s}$ $=1.2\left(e^{2} c / \pi \epsilon_{0} r_{b}^{2}\right) f\left[a_{w} F /\left(1+a_{w}^{2}\right)\right]^{2} \mathcal{Q}^{2}\left(\mathcal{E}_{b} / m c^{2}\right)^{2}$, where $\mathcal{Q}=1 / g \alpha$ is the quality factor of the cavity and $\mathcal{E}_{b}$ $=P_{e}\left(L_{b} / c\right)$ is the beam energy. The emission is superradiant, with the peak power proportional to the square of the beam current and the width inversely proportional to the square root of the beam current. Following the notation of Ref. [8] and introducing the high gain FEL parameter as $\rho=g_{0}^{1 / 3} / 4 \pi N_{w}$ and the cooperation length $L_{c}=\lambda / 4 \pi \rho$, the optimum efficiency and the peak power can be written respectively as $\eta=1.43 \rho \sqrt{\mathcal{Q} L_{b} / L_{c}}$ and $P_{s}$ $=1.2 \rho P_{e}\left(\mathcal{Q} L_{b} / L_{c}\right)^{2}$. These expressions relate the superradiant emission in high gain, single-pass FEL amplifiers to the superradiant emission in short pulse FEL oscillators [2,21]. Actually, in the first case the superradiant analysis has the same expressions for the efficiency and peak power, with $\mathcal{Q}=1$. If we image the emission in the perfectly tuned cavity as produced by a train of many electron pulses interacting with the radiation in the cavity for $n \sim \mathcal{Q}$ round-trips, then

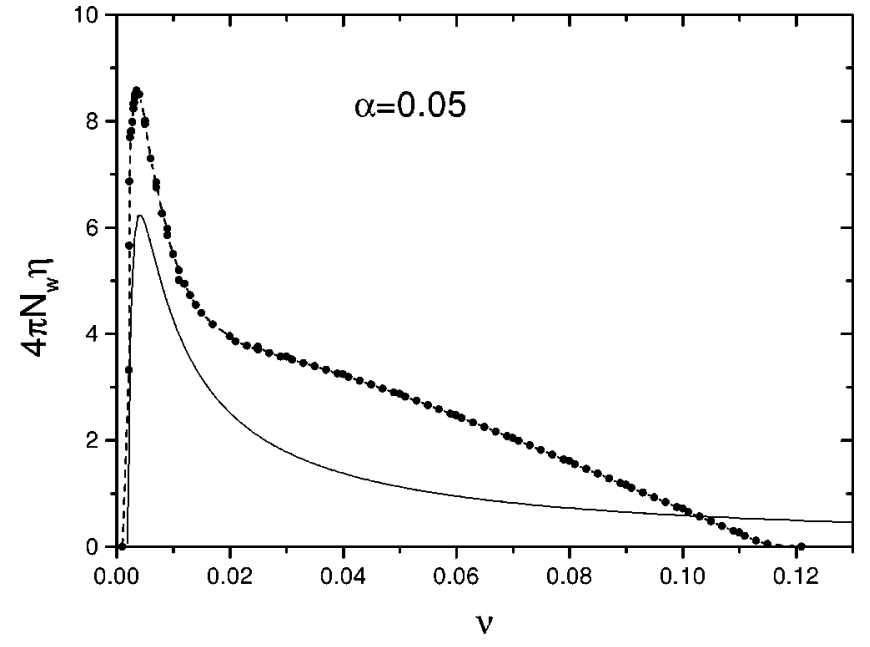

FIG. 10. Scaled efficiency $4 \pi N_{w} \eta$ vs $\nu$ for $\alpha=0.05$ from the numerical solution (circles) and from the analytical solution, Eq. (62) (solid line).

the emission is equivalent to the single-pass high gain interaction of the radiation with a beam of "effective" length $\mathcal{Q} L_{b}$.

In Fig. 10 we compare the efficiency for $\alpha=0.05$ vs $\nu$ as obtained from the numerical integration of Eqs. (3)-(7), with the expression (62) obtained under the assumption that for small cavity detuning $\nu$ all the eigenmodes are equal. For this value of $\alpha$, the amplitudes of the first two supermodes are almost equal, as may be seen in Fig. 8. The agreement with the superradiant limit is good enough near the peak at small $\nu$, considering that there are only two eigenvalues almost equal. Indeed we see from Eq. (30) that the eigenvalues $\mu_{n}$ become equal for $\nu \ll 2 /(n \pi)^{3}$, that is, for very small $\nu$ when $n \geqslant 3$. Unfortunately, decreasing $\alpha$ and $\nu$, the stationary superradiant solution is in general unstable (see Sec. VII) and the superradiant limit can be reached only in an average sense or in a narrow region near $\nu=0$ and $\alpha=0$.

\section{NONLINEAR MODES}

An important step in the study of a dynamical system is the determination of its stationary regimes. Time-dependent numerical simulations [11] have already shown that for cavity losses $\alpha$ and detuning $\nu$ in region 0 of the phase diagram Fig. 11, the system converges to a stable equilibrium. This region is limited on one side by the lasing threshold that has already been analyzed and on the other side by a limit cycle instability threshold. However, the time-dependent numerical simulations do not indicate what happens to the equilibrium at the limit cycle instability threshold or whether or not other stationary regimes exist.

In our case, it can be shown easily that the solutions of Eqs. (3)-(7) with constant amplitude are necessarily of the form

$$
A(x, \tau)=e^{i \omega \tau} A(x),
$$

where $\omega$ is some constant phase drift. Inserting Eq. (63) into Eqs. (3)-(7) with the appropriate boundary conditions, one gets the equations for the nonlinear modes 


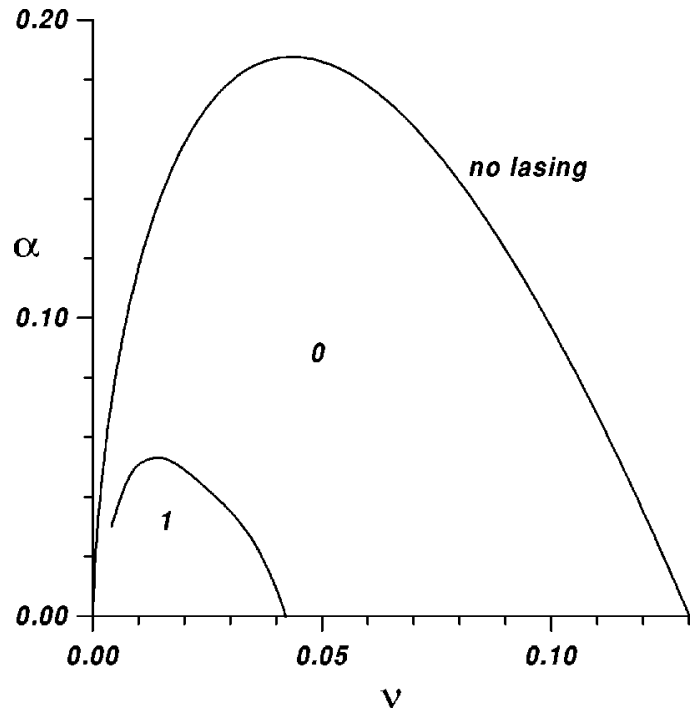

FIG. 11. Phase diagram giving the asymptotic behavior as a function of the operating parameters $\alpha$ and $\nu$. Region 0 , stable stationary regime; region 1 , limit cycle. For small $\alpha$ and $\nu$ see Fig. 15 .

$$
\begin{gathered}
\nu A^{\prime}=(i \omega+\alpha / 2) A-B, \\
B^{\prime}=-i P, \\
P^{\prime}=-A-i S B-2 i Q P+2 i Q^{2} B, \\
Q^{\prime}=-2 \operatorname{Re}\left(A B^{*}\right), \\
S^{\prime}=-4 \operatorname{Re}\left(A P^{*}\right), \\
x=0 \rightarrow B=P=Q=S=0, \\
x=1 \rightarrow A=0,
\end{gathered}
$$

where the prime represents the derivation with respect to $x$. Due to the global phase invariance, this mixed boundary value problem is overdetermined and has no solution in general, except for specific $\omega$ values. These $\omega$ values play the same role in our nonlinear stationary problem as the eigenvalues in the linear evolution problem. One way of finding (numerically) the $\omega$ 's and the corresponding nonlinear modes is as follows. For every value of the phase drift $\omega$ and for every $A_{0}$, one can solve the differential system Eqs. (64)-(68), from $x=0$ to $x=1$, with the initial conditions (69) and with a condition at the optical pulse head $A(0)=A_{0}$. The solution is an actual nonlinear mode if it also satisfies the boundary condition at the optical pulse tail Eq. (70). Now the value $A(1)$ at the tail is a function of the phase drift $\omega$ and the initial value $A_{0}$ : Let us note $A(1)=A_{1}\left(\omega, A_{0}\right)$. The search for nonlinear modes finally reduces to solving the nonlinear equation

$$
A_{1}\left(\omega, A_{0}\right)=0
$$

It seems hopeless to obtain closed form solutions of this problem. However, it can be handled numerically in the following way. First, due to the global phase invariance, we can restrict ourselves to $A_{0}$ real. Then the complex quantity $A_{1}\left(\omega, A_{0}\right)$ can be calculated on the $\left(\omega, A_{0}\right)$ plane by numeri-
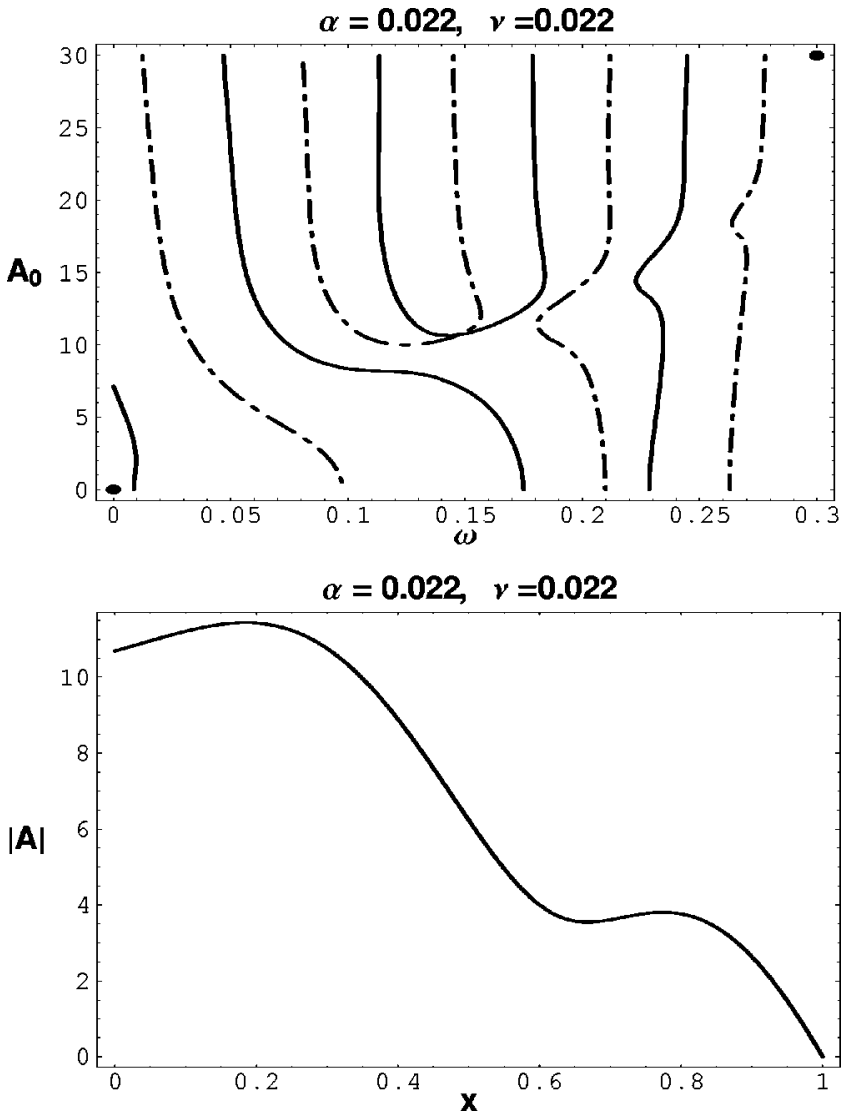

FIG. 12. Nonlinear mode for $\nu=\alpha=0.022$. Top: map $A_{1}\left(A_{0}, \omega\right)$; solid lines, $\operatorname{Re}\left(A_{1}\right)=0$; dash-dotted lines, $\operatorname{Im}\left(A_{1}\right)=0$. The amplitude $A_{0}$ at $x=0$ and the phase drift $\omega$ of the nonlinear mode are given by the intersection of solid and dash-dotted lines. Bottom: shape of the nonlinear mode.

cal integration of Eqs. (64)-(68). This allows one to find the level lines $\operatorname{Re} A_{1}=0$ and $\operatorname{Im} A_{1}=0$. The nonlinear modes are at the intersections of these two sets of lines.

Two examples are given in Figs. 12 and 13. Although for $\nu=\alpha=0.022$ the system does not evolve to an equilibrium but to a limit cycle (see Fig. 11), we find in the top part of Fig. 12 that an equilibrium still exists: Its phase drifts with $\omega \approx 0.15$ and its shape is given in the bottom part of Fig. 12 . This shape allows an exact compensation between lethargy and detuning, but is very different from the shape of the fundamental mode at the same $\nu=0.022$ : The optical field has a maximum close to the head, so the electrons strongly bunch in the first part of the wiggler.

In the second example, for $\nu=\alpha=0.015$ (see Fig. 13), we find two coexisting stationary solutions. Both their phases drift with $\omega \approx 0.15$. Although their shapes and amplitudes are quite different, they both realize an exact compensation between lethargy and detuning.

We give now a general picture of what is observed moving from large $\alpha$ and $\nu$ down to the more nonlinear regimes of smaller $\alpha$ and $\nu$. For large $\alpha$ and $\nu$, the only stationary regime is the basic state $A=0$ and it is stable: Large dissipation and fast transport out of the interaction region prevent laser amplification. The first nontrivial nonlinear mode, which we call the fundamental nonlinear mode, appears when the basic state $A=0$ loses its stability. This happens at the threshold $\alpha=2 \operatorname{Re} \mu_{1}(\nu)$, where $\mu_{1}(\nu)$ is the eigenvalue 

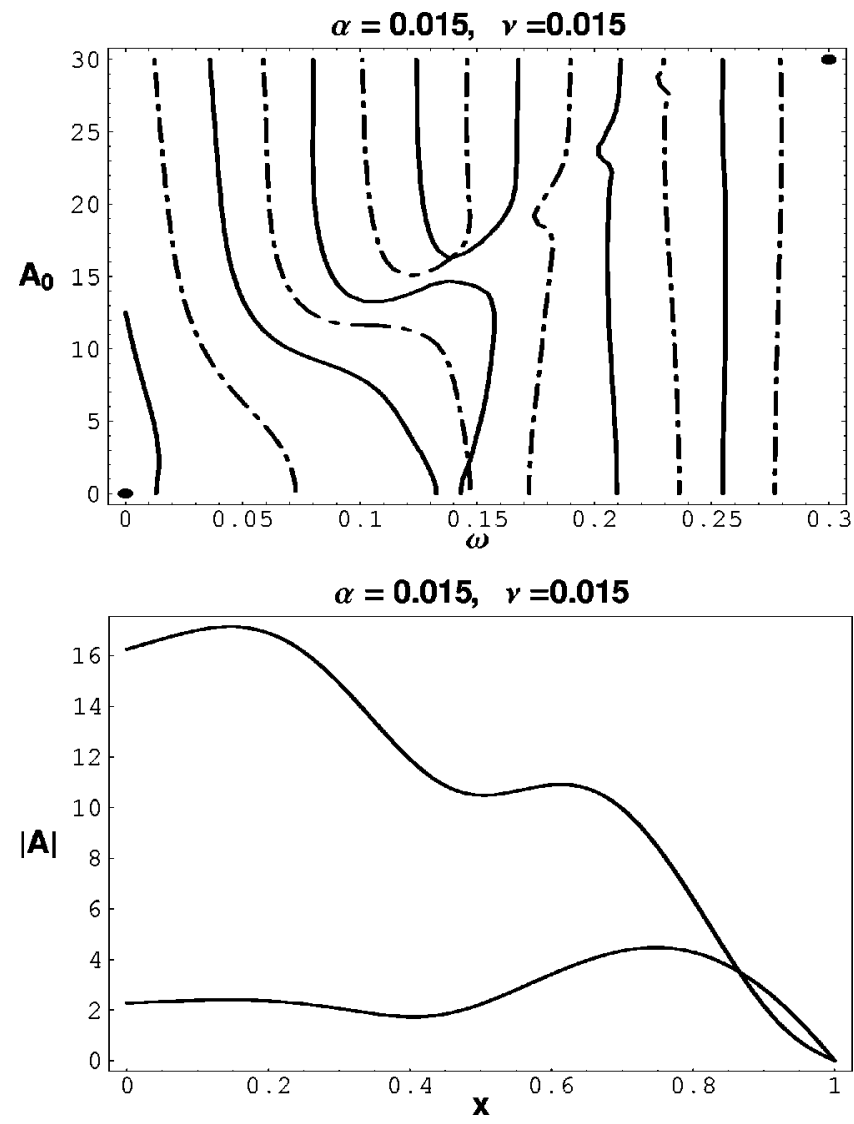

FIG. 13. Two coexisting nonlinear modes for $\nu=\alpha=0.015$. Top: map $A_{1}\left(A_{0}, \omega\right)$. Bottom: shapes of the nonlinear modes.

with the largest real part of the linearized problem. At this bifurcation point, $A_{0}=0$ and $\omega=\operatorname{Im} \mu_{1}(\nu)$. As long as one remains close to threshold, the nonlinear mode remains stable, small, and nearly proportional to the fundamental linear mode, as shown by the third-order study of the weakly nonlinear regime. Further from threshold, the nonlinear mode becomes larger, changes its shape, and eventually loses its stability (see Sec. VII), but continues to exist as observed in Fig. 12. At threshold for the second linear mode, i.e., for $\alpha=2 \operatorname{Re} \mu_{2}(\nu)$, where $\mu_{2}(\nu)$ is the eigenvalue with second larger real part, the basic state $A=0$ become again unstable in a second direction, corresponding to the second linear mode. A new bifurcation occurs and a new nontrivial nonlinear mode emerges, with $A_{0}=0$ and $\omega=\operatorname{Im} \mu_{2}(\nu)$, as shown by Fig. 13 (along the bisector of the phase diagram, the threshold for the second linear mode occurs at $\alpha=\nu$ $=0.0157)$. This nonlinear mode is itself unstable and will typically never be observed. In a similar way, a new bifurcation occurs at every threshold $\alpha=2 \operatorname{Re} \mu_{n}(\nu)$, leading to the emergence of a new unstable nonlinear mode close to the basic state $A_{0}=0$ and $\omega=\operatorname{Im} \mu_{n}$ and associated with the $n$th linear mode. Finally, at very small $\alpha$ and $\nu$, the system has an increasing number of (unstable) stationary regimes.

\section{LIMIT CYCLE INSTABILITY}

We consider now the stability of the fundamental nonlinear mode. We already know from experiments and previous numerical simulations [11] that for operating parameters $\nu$ and $\alpha$ decreasing away from the lasing threshold, one ob- serves successively the following behaviors.

The development of the limit cycle instability is exemplified in Fig. 14, which shows the evolution of the optical field amplitude profile $|A(x)|$ calculated from Eqs. (3)-(7) and the trajectory of the system in the phase space of the amplitudes of the first two linear modes ( $\rho_{1}$ and $\bar{a}_{2}$; see below). Close to the lasing threshold, the energy steadily increases up to saturation: When the optical field gets large enough, the electron beam cannot bunch proportionally any longer. Since the bunching is a cumulative effect along the wiggler, this saturation appears first at the wiggler exit (i.e., at the tail of the optical pulse $x \approx 1$ ). As a consequence, the gain drops at the tail of the pulse and the pulse peak slightly slips ahead (i.e., the lethargy drops). This slight forward deformation tends to increase the electron bunching and therefore compensates for the gain drop, thus leading to a steady saturated regime. See the right-hand side of Fig. 14(a).

Further from the lasing threshold, the system reaches saturation after damped oscillations: Just like before, saturation starts at the pulse tail $(x \approx 1)$. However, due to the smaller cavity detuning $\nu$, the transport towards the pulse head $(x \approx 0)$ of this nonlinear effect is slower and the gain at the tail drops under the cavity losses before the pulse has the time to slip ahead to compensate. Therefore, the optical field at the tail decreases. This perturbation is finally transported to the pulse head by the cavity detuning, leading to a slight increase of the bunching and the gain at the tail. The process is then repeated, producing damped oscillations propagating from the tail to the head of the optical pulse. See the righthand side of Fig. 14(b).

Still further from the lasing threshold, the system never reaches a stationary regime and evolves towards a limit cycle: Again, the gain drops first at the pulse tail, while the optical field still grows at the head, reaches a high level, and is finally evacuated forward by the cavity detuning, thus allowing a new start-up of the gain at the tail. The process is iterated, but the losses are now too low and the transport too slow to damp the oscillations and a limit cycle instead of a stationary saturated regime occurs. See the right-hand side of Fig. 14(c).

In order to understand these different behaviors, together with the fact that the fundamental nonlinear mode continues to exist, as shown by the analysis of Sec. VI, we reconsider the third-order two-mode weakly nonlinear approximation, introduced in Ref. [11]. Keeping the leading nonlinear terms for the first two modes in the approximation $\left|a_{2}\right| \ll\left|a_{1}\right| \ll 1$, Eqs. (27) and (46) reduce to

$$
\begin{gathered}
A(x, \tau)=a_{1}(\tau) A_{1}(x)+a_{2}(\tau) A_{2}(x), \\
\dot{a}_{1}=\left(\mu_{1}-\alpha / 2\right) a_{1}-\beta_{1}\left|a_{1}\right|^{2} a_{1}, \\
\dot{a}_{2}=\left(\mu_{2}-\alpha / 2\right) a_{2}-\beta_{2}\left|a_{1}\right|^{2} a_{1} .
\end{gathered}
$$

The solution of Eq. (73) is (up to a constant phase)

$$
\begin{gathered}
a_{1}=\rho_{1} e^{i \phi_{1}}, \\
\rho_{1}(\tau)=\frac{\rho_{1 s}}{\sqrt{1+\Gamma \exp \left(-g_{1} \tau\right)}},
\end{gathered}
$$


(a) $\quad \alpha=0.12, \nu=0.013$

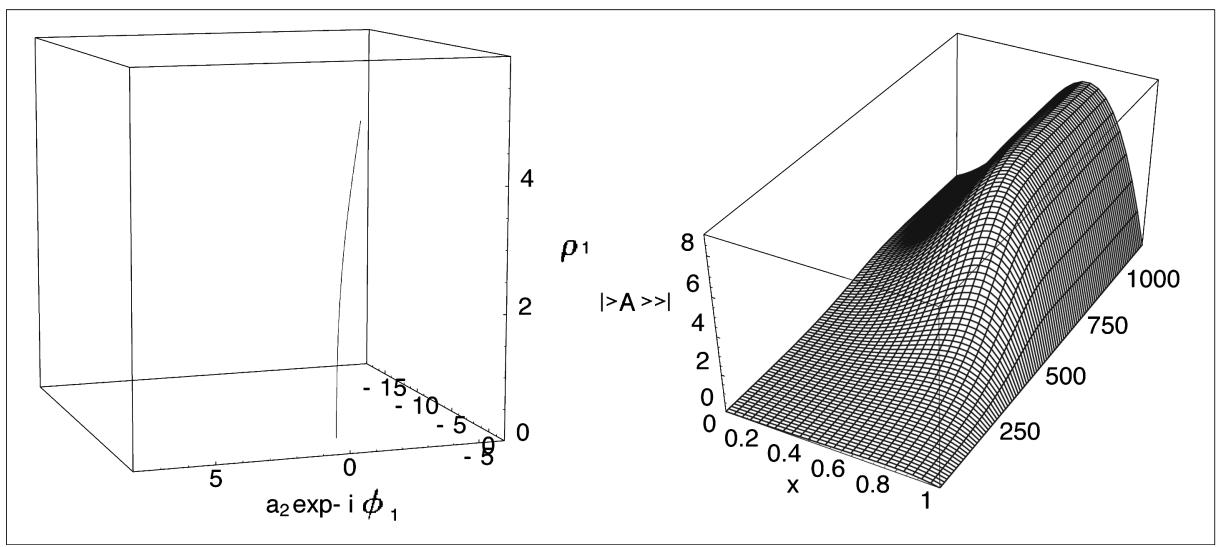

(b)

$\alpha=0.04, \nu=0.013$

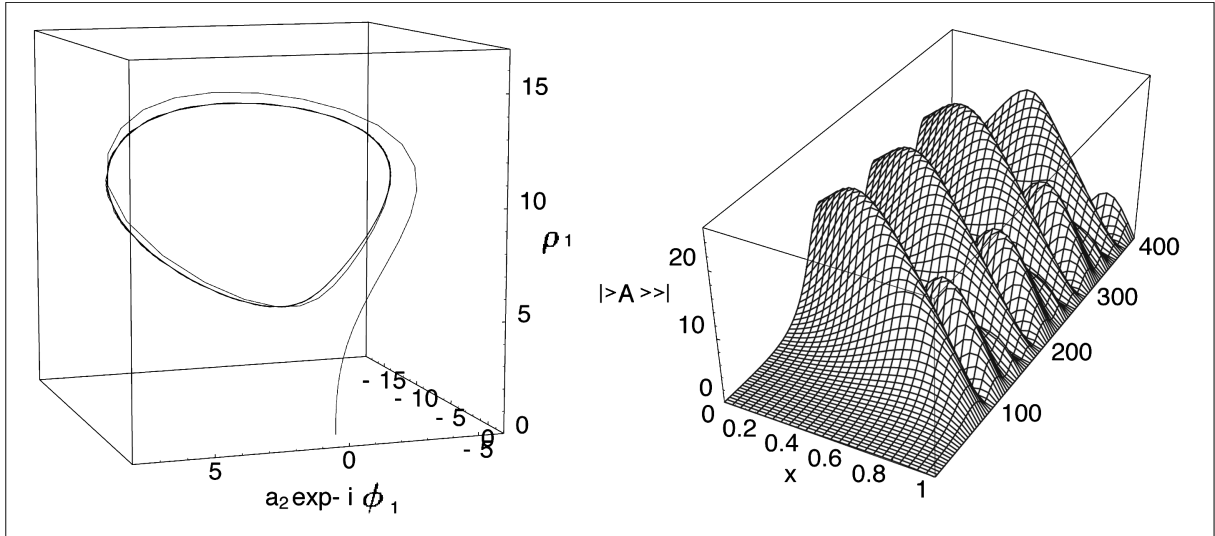

(c)

$\alpha=0.07, \nu=0.013$

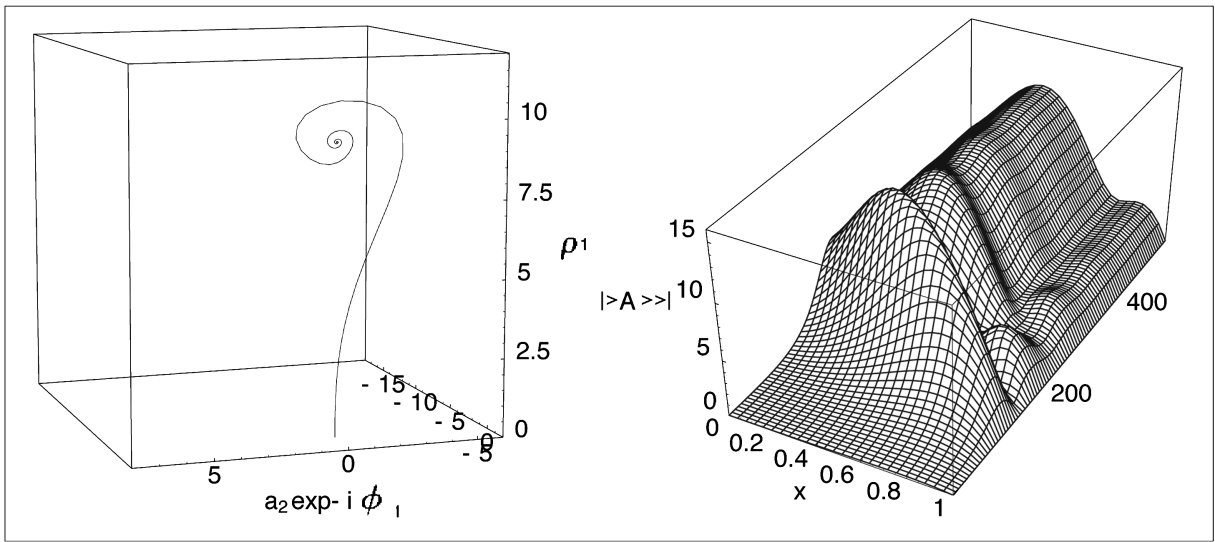

FIG. 14. Development of the limit cycle instability. On the right is the evolution of the optical field amplitude profile $|A(x)|$ calculated from Eqs. (3)-(7). On the left is the trajectory in the phase space of $\rho_{1}=\left|a_{1}\right|$ and $\bar{a}_{2}=a_{2} \exp \left(-i \phi_{1}\right)$. (a) Weakly nonlinear master-slave regime. (b) Damped oscillations to stable stationary regime. (c) Unstable equilibrium and limit cycle.

$$
\dot{\phi}_{1} \equiv \omega_{1}(\tau)=\operatorname{Im} \mu_{1}-\frac{\operatorname{Im} \beta_{1} \rho_{1 s}^{2}}{\sqrt{1+\Gamma \exp \left(-g_{1} \tau\right)}}
$$

where $\Gamma=\left(\rho_{1 s}^{2}-\rho_{10}^{2}\right) / \rho_{10}^{2}, \rho_{10}=\rho_{1}(0)$, and the saturated amplitude $\rho_{1 s}$, the phase drift $\omega_{1 s}$, and the net gain of the first two supermodes $g_{1,2}$ are given by Eqs. (47)-(49). Since a global phase of the system in irrelevant to the dynamics, it is useful to factor out the phase of the fundamental mode and write

$$
a_{2}=\bar{a}_{2} e^{i \phi_{1}}
$$

so that Eq. (74) becomes

$$
\dot{\bar{a}}_{2}=\left(\mu_{2}-i \omega_{1}-\alpha / 2\right) \bar{a}_{2}-\beta_{2} \rho_{1}^{3}
$$

If the second mode is strongly damped while the net gain of the fundamental is positive $\left(0<g_{1} \ll-g_{2}\right)$, we may assume 
the adiabatic approximation, which neglects the time derivative in Eq. (79), so that the second mode (slave) is driven by the fundamental (master)

$$
\bar{a}_{2}=\frac{\beta_{2} \rho_{1}^{3}}{\mu_{2}-i \omega_{1}-\alpha / 2},
$$

which at saturation goes to $\bar{a}_{2 s}$, given by Eq. (51). We observe, from Eqs. (76) and (80), a monotonic evolution of the system from low signal up to saturation. This behavior is observed on the left-hand side of Fig. 14(a), showing the trajectory of the system for $\alpha=0.12$ and $\nu=0.013$, in the three-dimensional phase space of the amplitudes $\rho_{1}, \operatorname{Re} \bar{a}_{2}$, and $\operatorname{Im} \bar{a}_{2}\left(\bar{a}_{2}\right.$ is complex and represents two degrees of freedom). The amplitudes $a_{1}$ and $a_{2}$ are calculated by Eq. (28). The net gains of the first two modes are $g_{1} \approx 0.013$ and $g_{2} \approx-0.095$, respectively.

If the net gain of the second mode is still negative but not much larger in amplitude than the net gain of the fundamental mode $\left(g_{2}<0<g_{1}\right)$, the evolution of $\bar{a}_{2}$ is no longer adiabatic. However, once the fundamental component has reached its stationary value, the $\bar{a}_{2}$ spirals down to the equilibrium $\bar{a}_{2 s}$ : We therefore observe damped oscillations before saturation. Indeed, a simple linear stability analysis of the system of equations (73) and (74) around the equilibrium $\rho_{1}=\rho_{1 s}$ and $\bar{a}_{2}=\bar{a}_{2 s}$ shows that it is stable as long as $g_{2}$ $<0$. This behavior is observed on the left-hand side of Fig. 14(b) for $\alpha=0.07$ and $\nu=0.013$. In this case the net gains of the first two modes are $g_{1} \approx 0.063$ and $g_{2} \approx-0.045$, respectively.

Finally, if the net gain of the second mode is not negative $\left(0<g_{2}<g_{1}\right)$, the equilibrium $\bar{a}_{2 s}$ is now the center of an unstable spiral. The asymptotic behavior of the system then depends on the nonlinearities in the vicinity of the equilibrium. Numerical simulations show that these nonlinearities stabilize the system, so we observe a small limit cycle around the unstable equilibrium. This description of the dynamics within the third-order two-mode approximation is rather crude, but it shows that the fundamental nonlinear mode becomes unstable via a supercritical Hopf bifurcation when pushing the operating parameters $\alpha$ and $\nu$ in the region where secondary linear modes can be excited. Note that in the nonlinear regime, the actual gain of the second mode is affected by the amplitude of the fundamental, so the limit cycle instability threshold is not accurately given by $g_{2}=0$ (i.e., $\alpha=2 \operatorname{Re} \mu_{2}$ ), but is already reached for $g_{2}<0$, so the domain of stability is actually smaller than expected from simple third-order two-mode approximation. This can be seen in Fig. 14(c) for $\alpha=0.04$ and $\nu=0.013$, where the net gains of the first two modes are $g_{1} \approx 0.093$ and $g_{2} \approx$ -0.0015 , respectively.

\section{ROUTE TO CHAOS}

The stability of the limit cycles themselves and the dynamical behavior for parameters $\alpha$ and $\nu$ going very close to zero have already been considered in $[10,11]$ within different approaches. Both period doubling and type-I intermittency [10] were observed in numerical simulations. We come again

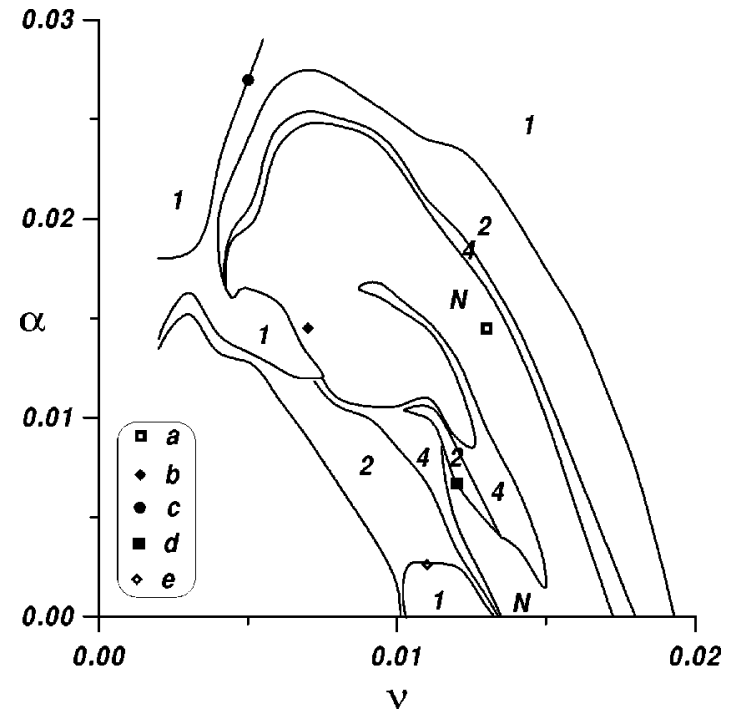

FIG. 15. Phase diagram giving the asymptotic behavior as a function of the operating parameters $\alpha$ and $\nu$ in the small $\alpha$ and $\nu$ region. Regions 1, limit cycle; regions 2, period-2 limit cycle; regions 4 , period-4 limit cycle; regions $N$ : chaos or period-largerthan-4 limit cycle; see the text and Fig. 16 for points a $(\nu$ $=0.013, \alpha=0.0145), \mathbf{b}(\nu=0.007, \alpha=0.0145), \mathbf{c}(\nu=0.005, \alpha$ $=0.027), \quad \mathbf{d}(\nu=0.012, \quad \alpha=0.0067), \quad$ and $\mathbf{e}(\nu=0.011, \quad \alpha$ $=0.0026)$.

to this point to show that other types of bifurcations also occur (basin boundary crossing, type-III intermittency), that the phase diagram, which is the separation of the parameter plane $(\alpha, \nu)$ into regions of definite asymptotic behavior, is much more intricate than previously stated, and finally that the dynamics can be described, to a large extent, by the mere iterations of a one-dimensional map. This description in terms of the iterations of a one-dimensional map is important because it allows us to identify the nature of the observed bifurcations in an easier and safer way. It may also be useful to devise future control strategies.

The results presented in this section are obtained by numerical integration of the model (3)-(7) and projection in the three-dimensional $\left(\bar{a}_{2}, \rho_{1}\right)$ phase space. They are summarized in Fig. 15, which shows the asymptotic behavior of the FEL depending on the reduced operating parameters $\alpha$ and $\nu$, starting from a low signal initial condition $A \approx 0$.

For decreasing $\alpha$ and $\nu$, one first recognizes a standard period doubling cascade (regions 1, 2, and 4) leading to high periodicity and chaotic regimes (region $N$ ). The essential features of this cascade of bifurcations are captured by the discrete dynamics of the successive maxima of the signal, given to a good approximation by the iterations of a onedimensional return map as shown in Fig. 16(a) for parameters given by the point a of Fig. 15. We observe that the attractor has the simple structure of a Rössler band [22], with a "one-bump" return map.

The phase diagram also presents other kinds of bifurcations, which can be also analyzed by plotting first return maps of the successive maxima of the amplitude $\rho_{1}$ of the fundamental mode. For example, point $\mathbf{b}$ in Fig. 15 is close to a bifurcation line between period-1 limit cycles and chaos. This is a subcritical period doubling bifurcation where the slope of the return map goes from slightly larger than -1 


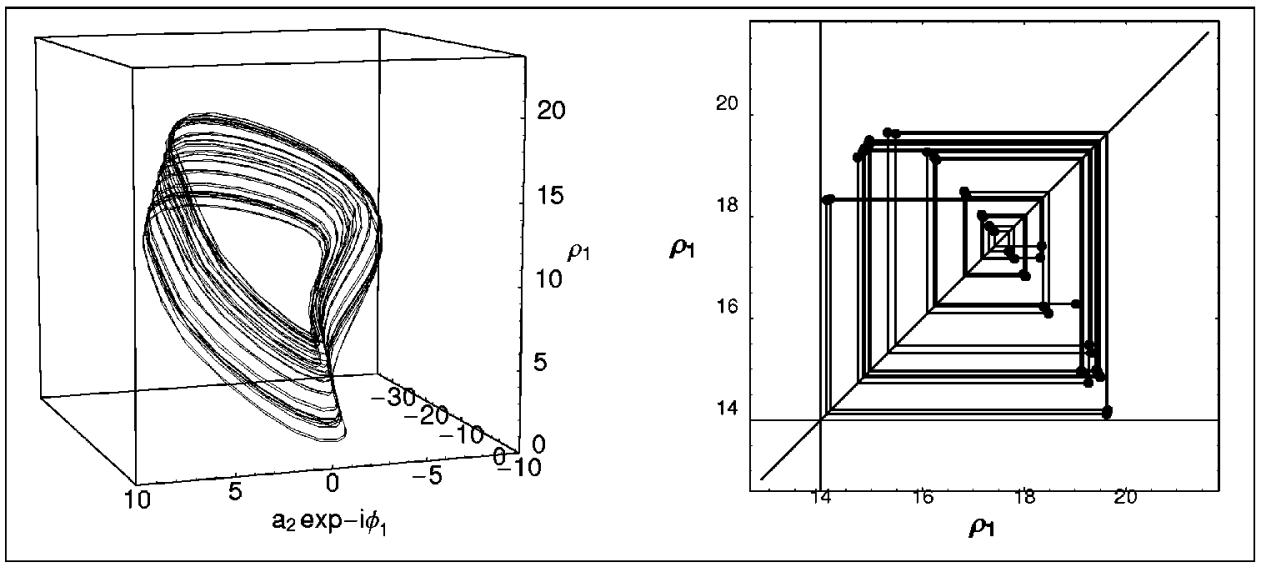

(b) $\alpha=0.0145, \quad v=0.007$
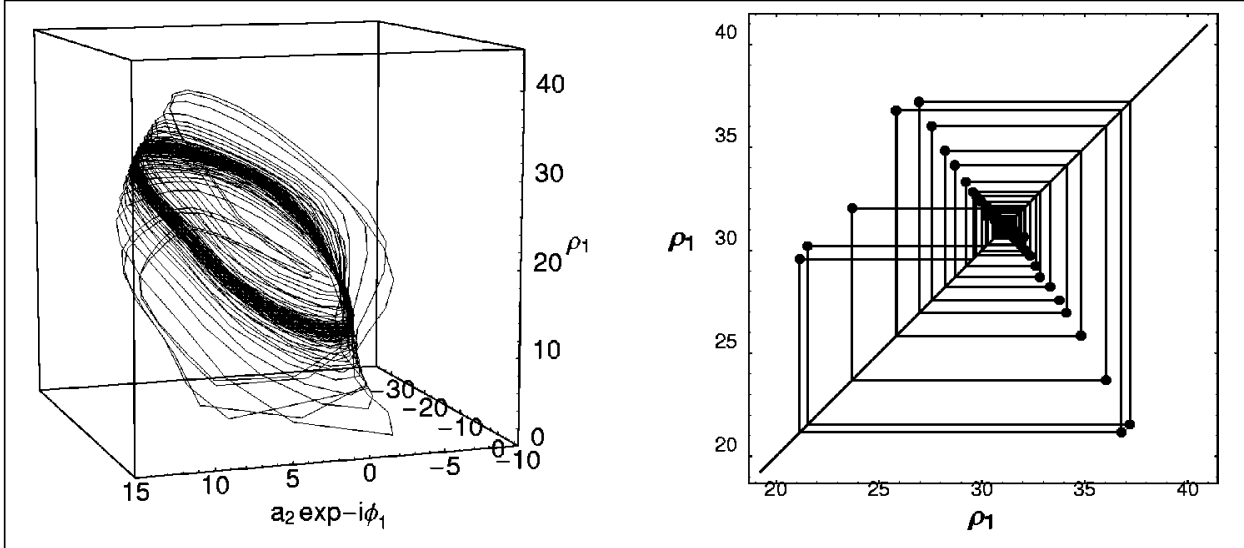

(c)

$\alpha=0.0265, \quad v=0.005$
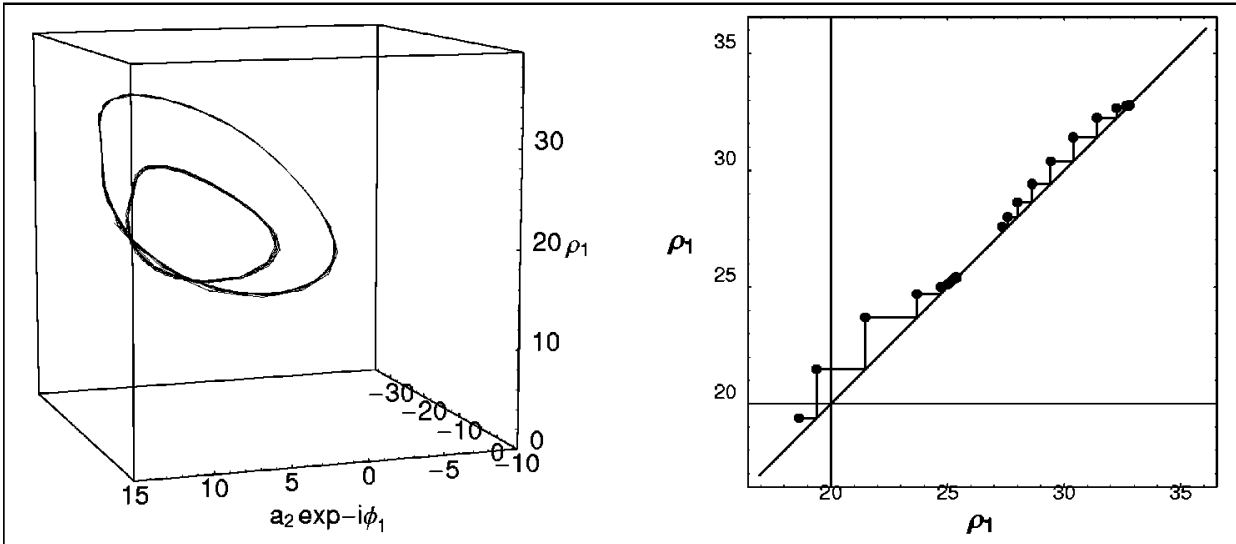

FIG. 16. Dynamics in the $\left(\rho_{1}, \bar{a}_{2}\right)$ amplitudes phase space and the corresponding return maps giving each maximum of the fundamental amplitude $\rho_{1}$ as a function of the previous one at (a) the chaotic point a of Fig. 15, close to the period doubling bifurcation cascade; (b) the chaotic point $\mathbf{b}$ of Fig. 15, close to the type-III intermittency subcritical bifurcation; and (c) the periodic point $\mathbf{c}$ of Fig. 15, where two period-1 limit cycles coexist, each one with its own basin of attraction.

(stable limit cycle of the system corresponding to a stable fixed point of the map) to slightly smaller than -1 (weakly unstable limit cycle and fixed point). This leads to type-III intermittency [23,24]: Nearly periodic episodes (close to the weakly unstable limit cycle) are separated by chaotic bursts (over the whole attractor). Each chaotic burst eventually brings the system back to the weakly unstable limit cycle, ready for a new nearly periodic episode. See Fig. 16(b).
Another type of bifurcation of the system occurs in the region of point $\mathbf{c}$ of Fig. 15, where two period-1 limit cycles coexist, each one with its own basin of attraction. Figure 16(c) shows, on the left-hand side, the two stable limit cycles corresponding to two stable fixed points of the return map and, on the right-hand side, the return map including the transients, the unstable fixed points (slope larger than 1), and the stable fixed points (positive slope smaller than 1). At 
point $\mathbf{c}$ and on the corresponding bifurcation line, the frontier between the basins of attraction of the two cycles sweeps over the low signal initial condition $A \approx 0$, which then changes from one basin to the other, thus leading to one cycle or the other. Note that in this region, slow changes of the operating parameters during the macropulse would lead to hysteresis phenomena. In a similar way, the bifurcation occurring at point $\mathbf{d}$ is a subcritical destabilization of a period-2 limit cycle, leading to a type-III intermittent chaos with period- 2 nearly periodic episodes separated by chaotic bursts, and the bifurcation occurring at point $\mathbf{e}$ is a basin boundary crossing with a coexistence of a period-1 limit cycle and a period-2 limit cycle.

\section{CONCLUSIONS}

In this paper we reconsidered the linear, weakly nonlinear, and nonlinear dynamics of short pulse free electron laser oscillators from a dynamical system point of view. The superradiant behavior appears in the limit of very small cavity detuning $\nu$, where all the supermodes converge and become nearly degenerate. In this sense, the superradiant regime in oscillators occurs when many supermodes of similar profile contribute to the total radiation field. In contrast, the singlesupermode regime occurs for relatively large $\alpha$, close to the fundamental supermode threshold $g_{1}=0$. We first showed that at small $\alpha$ and $\nu$, i.e., small cavity losses and detuning or high current, the system had several possible stationary solutions, but all unstable due to the "emancipation" of secondary modes. We found that the phase diagram (Figs. 11 and 15) of the system is quite intricated, making a fine control of the different dynamical behaviors in these devices difficult in practice. However, we also showed that the analysis of the transitions between the various possible unsteady regimes was made easier by the fact that the attractor of the system remains low dimensional, so that the dynamics on the attractor can be understood, to a large extent, in terms of iterations of one-dimensional return maps. This might help in the future for the design of control or stabilization schemes.
[1] Free Electron Lasers, edited by W.B. Colson, C. Pellegrini, and A. Renieri, Laser Handbook Vol. 6 (Elsevier, Amsterdam, 1990).

[2] D.A. Jaroszynski, P. Chaix, N. Piovella, D. Oepts, G.M.H. Knippels, A.F.G. van der Meer, and H.H. Weits, Phys. Rev. Lett. 78, 1699 (1997).

[3] N.M. Kroll, P.L. Morton, and M.N. Rosenbluth, IEEE J. Quantum Electron. QE-17, 1436 (1981).

[4] R.W. Warren, J.C. Goldstein, and B.E. Newnam, Nucl. Instrum. Methods Phys. Res. A 250, 19 (1986).

[5] D. Iracane, P. Chaix, and J.L. Ferrer, Phys. Rev. E 49, 800 (1994).

[6] D.A. Jaroszynski, R.J. Bakker, A.F.G. van der Meer, D. Oepts, and P.W. van Amersfoort, Phys. Rev. Lett. 70, 3412 (1993).

[7] R. Bonifacio, C. Maroli, and N. Piovella, Opt. Commun. 68, 369 (1988).

[8] R. Bonifacio, B.W.J. McNeil, and P. Pierini, Phys. Rev. A 40, 4467 (1989).

[9] H. Al-Abawi, F.A. Hopf, G.T. Moore, and M.O. Scully, Opt. Commun. 30, 235 (1979).

[10] S.J. Hahn and J.K. Lee, Phys. Lett. A 176, 339 (1993); Phys. Rev. E 48, 2162 (1993).

[11] N. Piovella, P. Chaix, G. Shvets, and D. Jaroszynski, Phys.
Rev. E 52, 5470 (1995).

[12] N. Piovella, P. Chaix, G. Shvets, and D.A. Jaroszynski, Nucl. Instrum. Methods Phys. Res. A 375, 156 (1996).

[13] D.A. Jaroszynski, D. Oepts, A.F.G. van der Meer and P. Chaix, Nucl. Instrum. Methods Phys. Res. A 407, 407 (1998).

[14] G. Dattoli, A. Marino, and A. Renieri, Opt. Commun. 35, 407 (1980).

[15] N. Piovella, Phys. Rev. E 51, 5147 (1995).

[16] R. Bonifacio, F. Casagrande, and L. De Salvo Souza, Phys. Rev. A 33, 2836 (1986).

[17] G. Shvets and J.S. Wurtele, Phys. Rev. E 56, 3606 (1997).

[18] Y.A. Bogomolov, V.L. Bratman, N.S. Ginzburg, M.I. Petelin, and A.D. Yunakovsky, Opt. Commun. 36, 209 (1981).

[19] G. Shvets (private communication).

[20] N.S. Ginzburg, S.P. Kuznetsov, and T.N. Fedoseeva, Radiophys. Quantum Electron. 21, 728 (1978).

[21] R. Bonifacio, N. Piovella, and B.W.J. McNeil, Phys. Rev. A 44, 3441 (1991).

[22] O.E. Rössler, Phys. Lett. 57A, 397 (1976).

[23] P. Manneville and Y. Pomeau, Physica D 1, 219 (1980).

[24] Y. Pomeau and P. Manneville, Commun. Math. Phys. 74, 189 (1980). 